\title{
The entrainment and energetics of turbulent plumes in a confined space
}

\author{
John Craske ${ }^{1} \dagger$ and Megan S. Davies Wykes ${ }^{2}$ \\ ${ }^{1}$ Department of Civil and Environmental Engineering, Imperial College London, \\ London SW7 2AZ, UK \\ ${ }^{2}$ Engineering Department, University of Cambridge, Trumpington Street, \\ Cambridge CB2 1PZ, UK
}

(Received xx; revised xx; accepted xx)

We analyse the entrainment and energetics of equal and opposite axisymmetric turbulent air plumes in a vertically confined space at a Rayleigh number of $1.24 \times 10^{7}$ using theory and direct numerical simulation. On domains of sufficiently large aspect ratio, the steady-state consists of turbulent plumes penetrating an interface between two layers of approximately uniform buoyancy. As described by Baines \& Turner ( $J$. Fluid Mech. vol. 37, 1969, pp. 51-80), upon penetrating the interface the flow in each plume becomes forced and behaves like a constant-momentum jet, due to a reduction in its mean buoyancy relative to the local environment. To observe the behaviour of the plumes we partition the domain into sub-domains corresponding to each plume. Domains of relatively small aspect ratio produce a single primary mean-flow circulation between the sub-domains that is maintained by entrainment into the plumes. At larger aspect ratios the mean flow between the sub-domains bifurcates, indicating the existence of a secondary circulation within each layer associated with entrainment into the jets. The largest aspect ratios studied here exhibit an additional, tertiary, circulation in the vicinity of the interface. Consistency between independent calculations of an effective entrainment coefficient allows us to identify aspect ratios for which the flow can be modelled using plume theory, under the assumption of a two-layer stratification.

To study the flow's energetics we use a local definition of available potential energy (APE). For plumes with Gaussian velocity and buoyancy profiles, the theory we develop suggests that the kinetic energy dissipation is split equally between the jets and the plumes and, collectively, accounts for almost half of the input of APE at the boundaries. In contrast, $1 / 4$ of the APE dissipation and background potential energy (BPE) production occurs in the jets, with the remaining $3 / 4$ occurring in the plumes. These bulk theoretical predictions agree with observations of BPE production from simulations to within $1 \%$ and form the basis of a similarity solution that models the vertical dependence of APE dissipation and BPE production. Unlike results concerning the dissipation of buoyancy variance and the strength of the circulations described above, the model for the flow's energetics does not involve an entrainment coefficient.

\section{Introduction}

The turbulent plume is a canonical buoyancy-driven flow that plays a fundamental role in a wide range of applications. Plumes induce flow (Taylor 1958) and produce a buoyancy structure (Worster \& Huppert 1983) in their surrounding environments, which in turn affects their behaviour. This coupling is of particular significance for applications

$\dagger$ Email address for correspondence: john.craske07@imperial.ac.uk 
involving confined spaces, such as the heating and ventilation of individual rooms in a building (Linden 1999), which are typically described using filling box (Worster \& Huppert 1983) and emptying filling box (Linden et al. 1990) models.

Current understanding of how a plume is affected by confinement is limited. Key questions in this regard relate to the effect of background turbulence, stratification, mean co- or cross-flow and entrainment or detrainment from nearby plumes (see e.g. Lai et al. 2019, Khorsandi et al. 2013, Gladstone \& Woods 2014, Bonnebaigt et al. 2018). All of these effects have the potential to influence the buoyancy structure of a space and the transport of pollutants. A particular challenge to research is that such effects typically coexist and each can influence the physics of plumes in several distinct ways. This makes it difficult to develop models using reductionism and might preclude simple explanations of observed phenomena. A case in point that has received attention recently is the observation and prediction that background turbulence reduces entrainment into turbulent jets (Hunt et al. 2006, Khorsandi et al. 2013, Lai et al. 2019), in spite of contrary suggestions elsewhere (Hubner 2006). A further example from the present study is our identification of a hierarchy of mean-flow structures (see Baines \& Turner 1969 , figure 10) induced by confined plumes, which are necessarily accompanied by background turbulence and a stable, layered stratification.

A central question concerning the various effects of confinement is their influence on entrainment, which is the closure for turbulence that underpins plume theory (Batchelor 1954, Morton et al. 1956). The entrainment coefficient determines the predicted ventilation and temperature of buildings (Linden 1999), in addition to the spreading rate of plumes (Morton et al. 1956). Recent work has therefore sought to establish a more precise and comprehensive understanding of the wealth of physics for which the entrainment coefficient implicitly accounts. The perspectives adopted range from studies of the turbulent/non-turbulent interface at the local microscale (van Reeuwijk \& Holzner 2014, da Silva et al. 2014; Burridge et al. 2017) to consistent relations with budgets for kinetic energy (Priestley \& Ball 1955; Kaminski et al. 2005; van Reeuwijk \& Craske 2015) and buoyancy variance (Craske et al. $\mid 2017$ ) from a global or integral perspective.

In spite of the leading role played by entrainment in relation to force (buoyancy) and flow (velocity) in jets and plumes, and their response to confinement, some results concerning energy budgets (involving the combination of force and flow) do not require a parameterisation of entrainment. An example is the stability properties of integral models of unsteady jets and plumes (Craske \& van Reeuwijk 2015b, 2016). A similar situation exists in relation to the Nusselt number and the mechanical energy budgets of RayleighBénard convection (Hughes et al.|2013). At high Rayleigh numbers, the energy budgets for Rayleigh-Bénard convection indicate that the input of available potential energy (APE) is partitioned equally between viscous dissipation and the energy required to homogenise the buoyancy field, which can be stated as the 'mixing efficiency' being equal to $1 / 2$. The finding contrasts with results pertaining to the dissipation of buoyancy variance per se (see e.g. Grossmann \& Lohse 2000), which depends on the Nusselt number and does not play a direct role in the system's energetics (Hughes et al. 2013). An identical result concerning the mixing efficiency of $1 / 2$ holds for a filling box regardless of the value of the entrainment coefficient (Davies Wykes et al. 2019). In contrast, the expression for the mixing efficiency of an emptying filling box depends, in a mathematical sense, on an entrainment coefficient (Davies Wykes et al. 2019).

Following Priestley \& Ball (1955), a connection between entrainment and a flow's budget for kinetic energy, the production of turbulence kinetic energy and, therefore, viscous dissipation is known (Kaminski et al. 2005, van Reeuwijk \& Craske 2015). Similar results, concerning the dissipation of buoyancy variance in plumes, are also known (Craske 


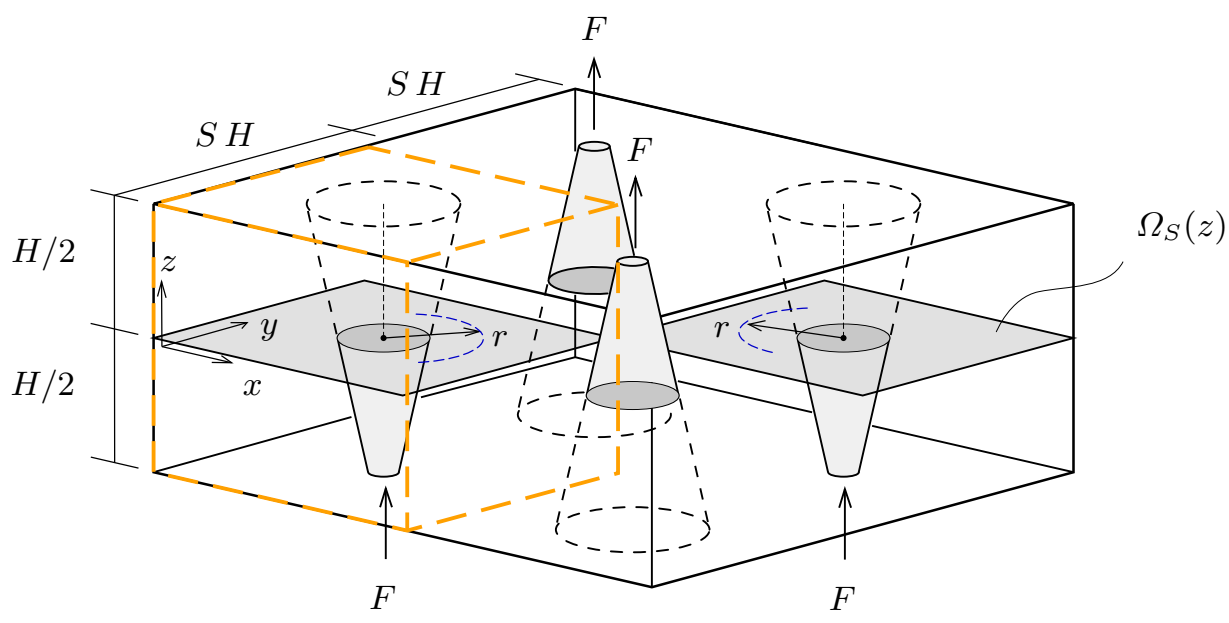

FIGURE 1. Schematic arrangement of four plumes within a horizontally periodic domain. The horizontal shaded regions comprising $\Omega_{S}(z)$ highlight parts of the horizontal domain over which integrals are taken. The dashed quadrant corresponds to the sub-domain to which discussion in the main text refers, comprised of a turbulent plume in the lower layer and, nominally, a turbulent jet in the upper layer. The domain height is $H$, the quadrant aspect ratio is $S$, and the buoyancy flux at the plume sources is $F$.

et al. 2017). However, as indicated in the preceding paragraph, buoyancy variance per se is not a quantity that plays a direct role in a system's energetics. To endow buoyancy variance with energetic implications, a system's APE must be considered (Winters et al. 1995), which necessarily introduces a mathematical dependence of the corresponding dissipative quantity on the global probability distribution for buoyancy.

Developments subsequent to Winters et al. (1995) that define local budgets of APE (Scotti et al. 2006; Roullet \& Klein 2009, Scotti \& White 2014; Novak \& Tailleux 2018), following Holliday \& Mcintyre (1981) and Andrews (1981), provide the opportunity for establishing a deeper understanding of energetics in the context of plume modelling. However, local APE frameworks for diagnosing stratified turbulence are still being developed and are relatively difficult to apply. For example, recent contributions from Scotti \& White (2014), Novak \& Tailleux (2018) and Tailleux (2018) propose alternative ways to partition mean and fluctuating components arising from turbulence.

An overarching aim of this work is to provide a bridge between the classical description of problems involving plumes in terms of entrainment and a deeper understanding of their energetics. In addressing this aim, we will clarify the differences between those aspects of confined convection that require a parameterisation for entrainment and those that can be deduced from arguments pertaining to the system's energetics alone. The latter are useful because they provide constraints on the flow's energy conversions. While we do not expect such constraints to restrict the value of the entrainment coefficient, we expect the information to be valuable more generally in the development and understanding of bulk models for plumes in confined spaces. Our hope is that the link we provide with the established global energetics framework of Winters et al. (1995) will elicit application of the local APE frameworks described in the previous paragraph to diagnose aspects of the small-scale physics associated with entrainment.

The flow we consider is driven by equal and opposite point sources of buoyancy, as 
shown in figure 1. The case provides a convenient means of addressing several of the questions outlined above, such as those relating to the interaction of the plumes with each other and with a step change in ambient buoyancy (Baines \& Turner 1969, Camassa et al. 2016). Indeed, the case is complementary or dual to the approximately uniform mean buoyancy produced in Rayleigh-Bénard convection, and therefore provides a useful setting to investigate the flow's energetics in relation to previous work (Hughes et al. 2013, Davies Wykes et al. 2019).

After describing the problem and simulation details in $\$ 2$, we structure the paper as two parts. The first (\$3) deals with the buoyancy and flow structure, which are aspects of the problem that relate explicitly to entrainment. The second (\$4), in contrast, demonstrates that the flow's energetics can be understood without reference to entrainment.

\section{Problem definition}

\subsection{Overview}

The case that we examine consists of an array of four plumes in a horizontally periodic and square domain of height $H$ and horizontal dimensions $2 S H \times 2 S H$, as shown in figure 1. Each diagonal pair of plumes is driven by sources of buoyancy on either the top or the bottom of the domain. The sources are located in the centre of each quadrant and each provides a positive buoyancy flux $F$. For sufficiently large $S$, the resulting steady state consists of a stable two-layer stratification with a step change in buoyancy that is determined by entrainment into the plumes. An equivalent case was described in terms of line and point sources by Baines \& Turner (1969, p.72).

Figure 2 displays a vertical slice through the instantaneous buoyancy field for $S=1$. The buoyancy structure produced by localised sources of buoyancy contrasts with the approximately uniform state that would be produced by the uniform heating and cooling of the horizontal boundaries for Rayleigh Bénard convection. In the lower layer above a heat source a turbulent plume develops, whose fluid penetrates the interface between the layers, before being entrained by the adjacent plumes.

\subsection{Governing equations}

The equations of motion for the Boussinesq flow considered here are

$$
\begin{aligned}
\frac{\partial \boldsymbol{u}}{\partial t}+\boldsymbol{u} \cdot \nabla \boldsymbol{u} & =-\nabla p+b \boldsymbol{k}+\frac{1}{R e} \nabla^{2} \boldsymbol{u}, \\
\nabla \cdot \boldsymbol{u} & =0 \\
\frac{\partial b}{\partial t}+\boldsymbol{u} \cdot \nabla b & =\frac{1}{P e} \nabla^{2} b,
\end{aligned}
$$

in which the scales used to non-dimensionalise velocity $\boldsymbol{u}$, buoyancy $b$, kinematic pressure $p$, space $(x, y, z)$ and time $t$ are $F^{1 / 3} H^{-1 / 3}, F^{2 / 3} H^{-5 / 3}, F^{2 / 3} H^{-2 / 3}, H$ and $F^{-1 / 3} H^{4 / 3}$, respectively. The unit vector $\boldsymbol{k}$, points in the vertical direction. The Reynolds and Péclet numbers are $R e=F^{1 / 3} H^{2 / 3} / \nu$ and $P e=F^{1 / 3} H^{2 / 3} / \kappa$, respectively, for kinematic viscosity $\nu$ and thermal diffusivity $\kappa$. In terms of dimensionless variables, the buoyancy flux supplied by each source is equal to unity. To estimate an integral time scale for this problem we divide the quadrant volume $H^{3} S^{2}$ by the volume flux $F^{1 / 3} H^{5 / 3}$, which results in a horizontal turnover time of $F^{-1 / 3} H^{4 / 3} S^{2}$. 


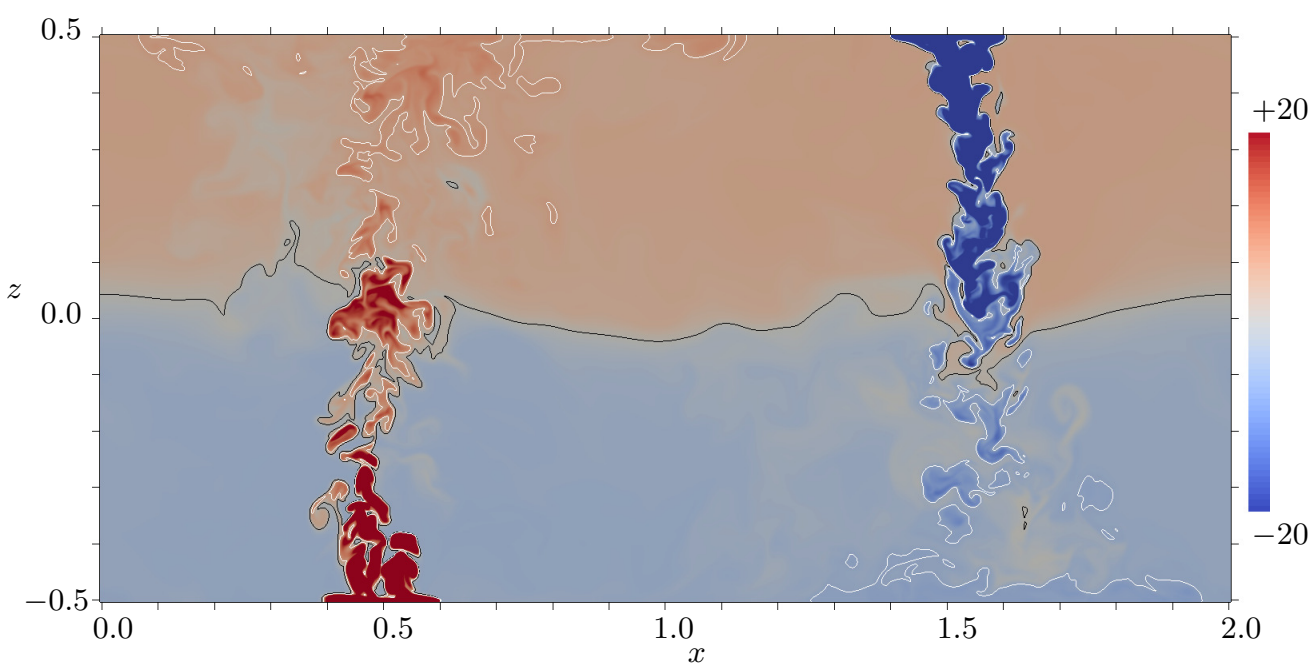

FIgURE 2. The buoyancy field $b(x, z)$ over a vertical slice of the domain. The domain shown has a quadrant aspect ratio of $S=1$ and the slice intersects the vertical axis of two of the plumes. The black line denotes a buoyancy isosurface of zero and the two white lines denote buoyancy isosurfaces at \pm 5.6 .

\subsection{Simulation details}

We will compare theoretical predictions to the direct simulation of 2.1$)-(2.3)$. The code we use employs a fourth-order finite volume discretisation, the details of which are documented in Craske \& van Reeuwijk (2015a). We simulate the medium of air, with a Prandtl number $\operatorname{Pr}=\nu / \kappa=0.71$ and consider flows with Reynolds number $R e=4185$, which corresponds to a Rayleigh number of $\operatorname{Pr} \operatorname{Re}^{2}=1.24 \times 10^{7}$. On the horizontal (top and bottom) boundaries we apply a free-slip condition on the velocity and homogeneous Neumann conditions on the buoyancy field outside the circular sources. Inside the circular sources we impose an inhomogeneous Neumann condition on buoyancy to produce a constant flux. On the vertical boundaries (sides) of the domain we impose periodic boundary conditions on all dependent variables. The grid is Cartesian and uniformly spaced; hence the shape of the nominally circular sources is formed approximately using square cells to fill a disk of diameter $D=H / 5$. The plumes are unforced and therefore nominally lazy at their source (Hunt \& Kaye 2005). This means that the plumes contract in the vicinity of the source (Fanneløp \& Webber 2003), resulting in an effective radius that is significantly less than the physical source radius (see, e.g. the sources in figure 2). A summary of the simulation details is provided in table 1 and in appendix $B$ we demonstrate the convergence of the results and investigate their sensitivity with respect to changes in the Reynolds number.

\subsection{Domain decomposition}

We take statistics over quadrants that are centred on each source, as illustrated in figure 1. Unlike the statistics that can be obtained over the entire horizontal plane, the quadrant statistics are, in general, vertically asymmetric with respect to the interface and allow us to isolate the vertical evolution of each plume. In addition, it proves convenient to respect the symmetry of the problem by aligning the origin of the vertical coordinate $z$ with the interface, as shown in figure 1 .

We define time-averaged integrals by integrating over diagonal pairs of quadrants 


\begin{tabular}{rrrr} 
& $N x \times N y \times N z$ & \multicolumn{1}{c}{$S$} \\
$\star$ & $768^{2} \times 768$ & $1 / 2=0.50$ \\
$\bullet$ & $896^{2} \times 768$ & $7 / 12$ & $=0.58$ \\
$\square$ & $960^{2} \times 768$ & $15 / 24$ & $=0.63$ \\
$\diamond$ & $1024^{2} \times 768$ & $2 / 3$ & $=0.67$ \\
$\triangle$ & $1536^{2} \times 768$ & 1 & $=1.00$ \\
$\bullet$ & $2048^{2} \times 768$ & $4 / 3$ & $=1.33$
\end{tabular}

TABle 1. Simulation details. All simulations were run at $R e=4185, P e=2971$, and $D N_{x} / 2 S H=154$, where $D N_{x} / 2 S H$ is the number of cells that span the source diameter $D$ for quadrant aspect ratio $S$. The symbols in the leftmost column correspond to those used in figures hereafter.

$\Omega_{S}(z)$, for which the flow is statistically equivalent (see, for example, the square shaded regions in figure 1), such that

$$
\langle f\rangle(z) \equiv \frac{1}{2 T} \int_{0}^{T} \iint_{\Omega_{S}(z)} f(\boldsymbol{x}, t) \mathrm{d} x \mathrm{~d} y \mathrm{~d} t,
$$

The time $T$ over which the integrals were averaged was not less than 1 dimensionless horizontal turnover time $F^{1 / 3} H^{-4 / 3} S^{2}$. Whilst the factor of $1 / 2$ in 2.4 means that $\langle f\rangle$ can be interpreted as an integral over a 'single' quadrant, we emphasise that 2.4 integrates information from a diagonal pair of quadrants, in which the flow has the same sense, as shown in figure 1.

We will use double angles to denote integrals over a volume of the domain,

$$
\langle\langle f\rangle\rangle_{a}^{b} \equiv \int_{a}^{b}\langle f\rangle(z) \mathrm{d} z .
$$

Acknowledging that our use of \langle\rangle and $\langle\langle\rangle\rangle$ to denote integrals rather than averages is not standard, we note that for this problem, in which we vary the aspect ratio, it proves convenient. For example, the constant area-integrated buoyancy flux at each plume source is independent of a domain's aspect ratio, in contrast to the spatially averaged buoyancy flux, which is inversely proportional to the aspect ratio squared.

\section{Mean flow and buoyancy structure}

In this section we discuss the flow's mean velocity and buoyancy structure in relation to the effects of entrainment and observe their dependence on the domains' aspect ratio.

\subsection{Observations}

Convection above or below each source in figure 2 results in turbulent plumes that entrain, dilute and transport fluid between the layers and, in doing so, determine an approximately two-layer stratification of the surrounding environment (Baines \& Turner 1969). Buoyancy conservation indicates that in a statistically steady state, in which the ambient buoyancy in each layer does not change, the mean buoyancy in a plume at the level of the interface is approximately equal to the ambient buoyancy of the layer downstream. We will therefore refer to the resulting neutrally buoyant flow into the 
(a)

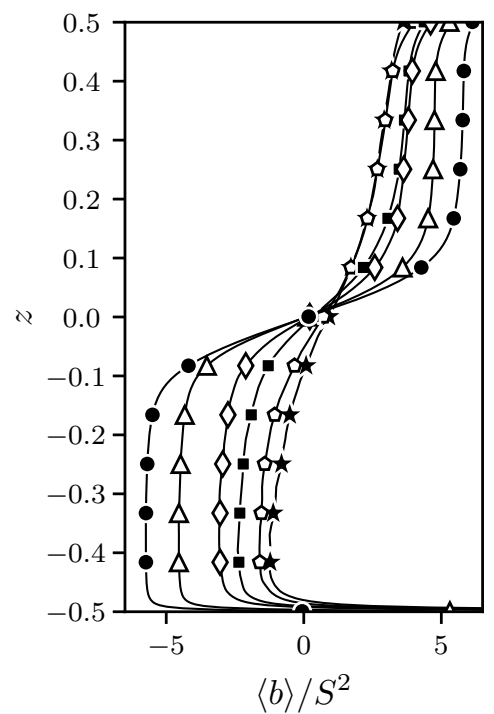

(b)

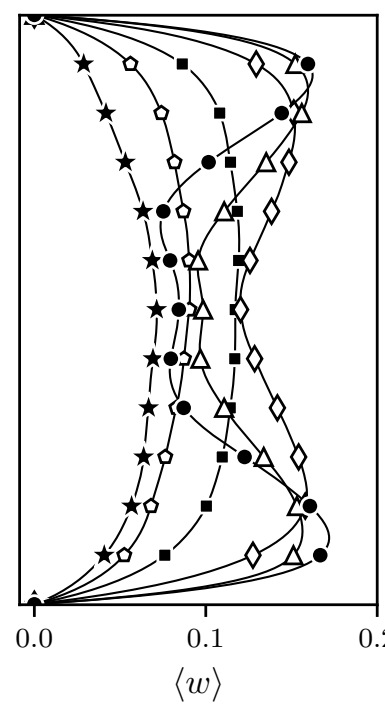

(c)

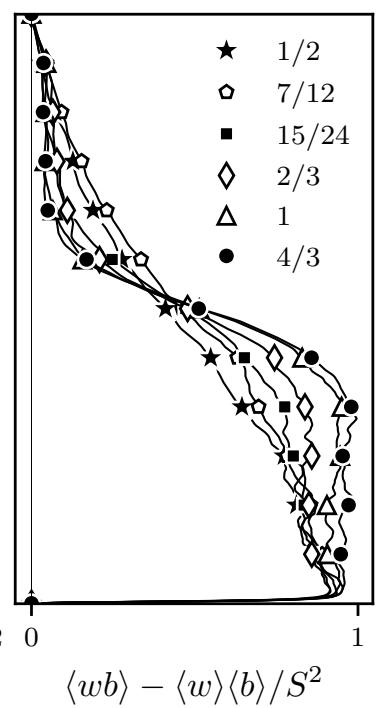

Figure 3. Quadrant integrals of the average ( $a$ ) buoyancy (divided by $S^{2}$ ), (b) volume flux, and (c) relative buoyancy flux, for aspect ratios $S=1 / 2,7 / 12,15 / 24,2 / 3,1$, and $4 / 3$, as indicated by the symbols. The plume occupies the lower half of the domain, as highlighted in figure 1

downstream layer as a turbulent jet (as can be seen in the top left and bottom right of figure 2. For discussion purposes we will focus on the quadrant containing a plume oriented in the positive vertical direction, as highlighted in figure 1, and will therefore refer to the plume as occupying the lower layer and the jet as occupying the upper layer. In spite of the terminology we employ, we can see in figure 1 that density anomalies persist within the jets, rendering their flow different from conventional uniform-density jets.

Figure 3 indicates how quadrant integrals of buoyancy, volume flux and relative buoyancy flux vary in the vertical direction. Figure $3(a)$ shows that as the aspect ratio of the domain increases, the difference between the buoyancy of the lower and upper layer increases. The buoyancy profiles in figure $3(a)$ are slightly asymmetric due to the influence of the plume, whose mean buoyancy is non-zero, in the lower layer. The asymmetry is relatively weak because the volume occupied by the plume is small in comparison with that of the domain.

The vertical volume flux is zero when integrated over the entire domain, but nonzero when integrated over a single quadrant. By examining the behaviour of the vertical volume flux in a single quadrant, we can therefore identify the strength and structure of the large-scale circulation. For example, if the vertical volume flux $\langle w\rangle$ is increasing with height $z$ then, by continuity, fluid is being drawn in through the sides of the quadrant at that height $z$. If, on the other hand, the vertical volume flux is decreasing with height, then there is a net flow of fluid out of the quadrant through its sides.

The vertical volume flux for a single quadrant is plotted in figure $3(b)$. For the three smallest aspect ratios $S=1 / 2,7 / 12,15 / 24$, figure $3(b)$ shows that the volume flux increases in a quadrant above the source (located at $z=-0.5$ in figure 3), before decreasing in the upper layer. This indicates that fluid is drawn into the quadrant through the sides in the lower layer, transported vertically between the layers and subsequently transported out of the quadrant through the sides in the upper layer, as evidenced 
(a)

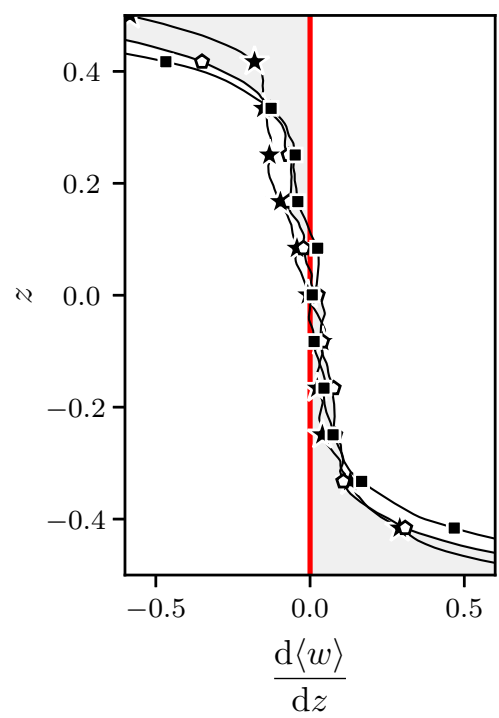

(b)

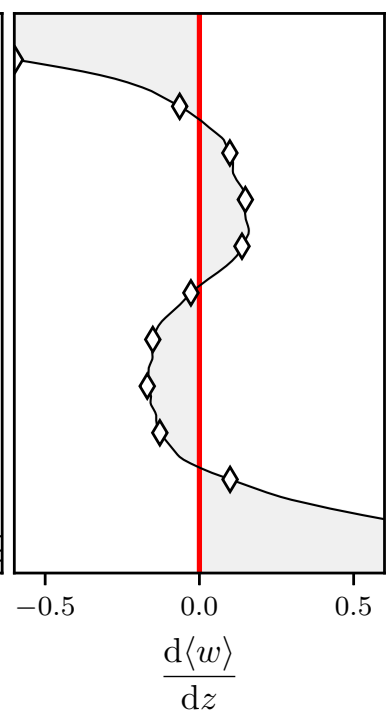

$(c)$

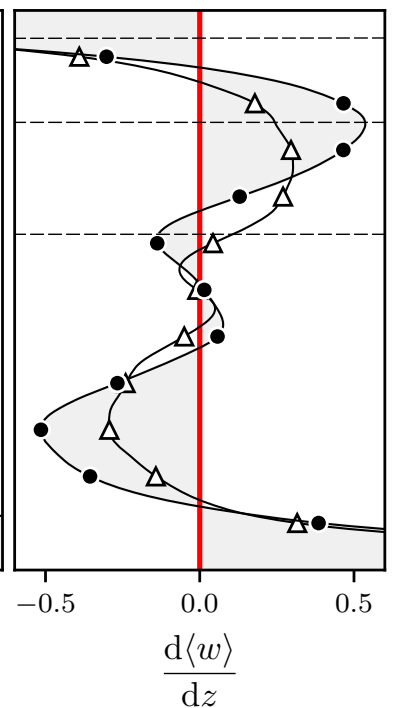

FIgURE 4. The vertical derivative of the quadrant volume flux $\langle w\rangle$, which is equal to the horizontal flow through the vertical sides of the quadrant. The flow on domains of aspect ratio $S=1 / 2,7 / 12,15 / 24$ are shown in $(a)$ and do not contain any critical points. The flow on a domain of aspect ratio $S=2 / 3$ is shown in $(b)$ and contains two critical points and therefore bidirectional flow through the vertical sides of the quadrant in the upper and lower half of the domain. Flows on domains of aspect ratio $S=1,4 / 3$ are shown in (c) and contain four critical points, indicating the presence of a tertiary circulation cell at the interface. Horizontal cross-sections of the velocity field are plotted for the $S=4 / 3$ case in figure 5 at the heights indicated by dashed lines on $(c)$.

explicitly in figure $4(a)$, which displays the vertical derivative of the volume flux. From the perspective of the spatially averaged flow, the entrainment and convection driven by the plumes results in a single-cell large-scale circulation between the quadrants, and volume conservation implies that these processes are necessarily symmetric about the mid-plane $z=0$.

In a spatially averaged sense, a topological change in the flow between quadrants occurs when the aspect ratio increases to $2 / 3$. This can be identified from the emergence of two local maxima in $\langle w\rangle$, as seen in figure $3(b)$, and the bidirectional flow in the upper and lower halves of figure $4(b)$. At a local maximum in $\langle w\rangle$, the mean horizontal flow across the sides of the quadrant changes direction, from a net flow into the quadrant to a net flow out of the quadrant. The appearance of two additional maxima therefore indicates that secondary circulation cells have developed in each layer. Noting that secondary circulations might exist within, rather than between, each quadrant for $S \leqslant 15 / 24$, we conclude that $S>15 / 24$ entails a secondary circulation that engulfs more than half of a given layer.

The secondary circulation cells form due to entrainment into the turbulent jets. When the plumes are sufficiently far apart, a given jet entrains fluid in addition to that which is supplied by the plume; hence the flux of volume that leaves the quadrant where the jet impinges on the horizontal boundary of the domain exceeds the total volume entrained by the plumes in the neighbouring quadrants and the residual volume recirculates. The existence of secondary circulation cells is therefore an indication that the plumes are, 
to some extent, behaving independently. We will demonstrate in $\$ 4.6$, where we analyse the velocity profiles within the jet, that the residual volume flux is indeed driven by entrainment into the jet, rather than being sustained as a co-flow outside the jet, which is not assured by the information in figures 3 and 4 .

The additional volume flux due to entrainment into the jet can be calculated using plume theory. We assume that the flow nominally behaves as a turbulent jet above the interface. More precisely, the flow is a forced plume, because figure $3(c)$ indicates a residual buoyancy flux in the flow above the interface, which diminishes with aspect ratio. The volume flux in the jet is equal to the volume flux in the plume at the interface plus the volume flux due to the subsequent entrainment into the jet,

$$
Q(z)=\underbrace{Q_{m}}_{\text {plume }}+\underbrace{\frac{6 \alpha \sqrt{\pi}}{5} M_{m}^{1 / 2} z}_{\text {jet }}
$$

where $\alpha$ is the entrainment coefficient for a plume and we have explicitly accounted for the fact that the entrainment coefficient in plumes is approximately $5 / 3$ larger than it is in jets (van Reeuwijk \& Craske 2015). Hereafter it proves convenient to label the distance from the plume source to the interface using the symbol $\zeta$. Hence, the quantities $Q_{m}$ and $M_{m}$ in (3.1), corresponding to the volume flux and momentum flux in the plume at the interface, respectively are,

$$
Q_{m}=\frac{6 \alpha}{5}\left(\frac{9 \alpha \pi^{2}}{10}\right)^{1 / 3} \zeta^{5 / 3}, \quad M_{m}=\pi^{1 / 3}\left(\frac{9 \alpha}{10}\right)^{2 / 3} \zeta^{4 / 3} .
$$

Equations $(3.2 a)$ and $(3.2 b)$ assume that the local (lower layer) environment is unstratified; a stratification would result in values of $Q_{m}$ and $M_{m}$ less than those predicted by $(3.2 a)$ and $(3.2 b)$. A comparison of equation $(3.1)$ at $z=0$ with equation $3.2 a)$ indicates that the rate at which the jet entrains volume $Q(z=\zeta)-Q_{m}$ is equal to the total volume entrainment rate $Q_{m}$ of the plume. This property is a consequence of the observed ratio of $5 / 3$ between the entrainment coefficient in a plume compared with a jet (van Reeuwijk \& Craske 2015). Consequently, for sufficiently large aspect ratios, the strength of the secondary circulation is equal to the strength of the primary circulation, as can be verified from figure $3(b)$, which shows that the maximum value of $\langle w\rangle$ is twice as large as its value at $z=0$ for $S=4 / 3$. The maximum volume flux in a quadrant results from entrainment into the plume in addition to fluid that will be re-entrained by the adjacent jet.

For the domains of relatively large aspect ratio, such as $S=1$ and $S=4 / 3$, figure $3(b)$ shows that a further bifurcation in the mean-flow structure occurs. The change corresponds to the emergence of a third, relatively weak, circulation cell, which can also be identified from the two additional critical points in $\langle w\rangle$, as shown in figures $3(b)$ and $4(c)$. The three circulation cells for $S=4 / 3$ can also be observed in the horizontal velocity fields shown in figure 5. The quadrants for which the integrals in figures 4 and 5 were calculated correspond to the bottom left and top right quadrants. Just above the interface, where $z=0.1$, there is a net inflow into the top left and bottom right quadrants and a net outflow from the top right quadrant. At $z=0.3$, the divergence is stronger and indicates that the flow entering or leaving a given quadrant has reversed in comparison with $z=0.1$. At $z=0.45$ the direction of flow entering and leaving each quadrant changes for a second time and corresponds to the primary circulation driven by entrainment into the plumes.

We close our analysis of the mean flow's topology by noting a likely influence of free- 
(a)

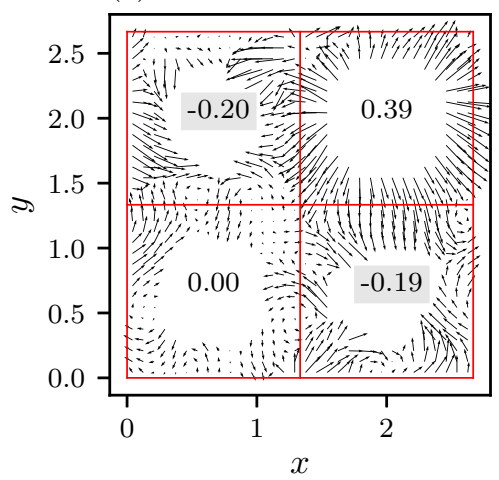

(b)

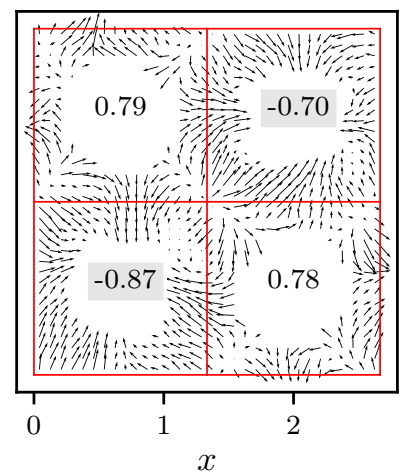

$(c)$

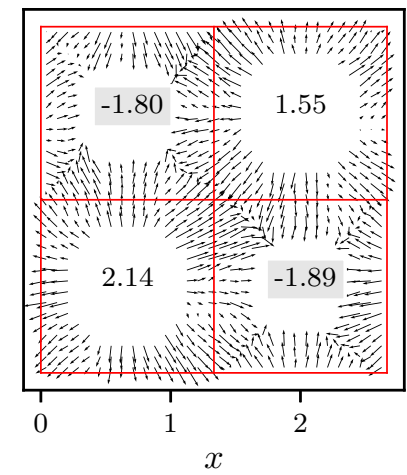

Figure 5. Horizontal slices through the time-averaged velocity field and the horizontal divergence $\left\langle\partial_{x} u+\partial_{y} v\right\rangle$ (displayed in text) at $(a) z=0.1,(b) z=0.3,(c) z=0.45$ for aspect ratio $S=4 / 3$. The locations of these slices are plotted as dashed lines on figure $4(c)$. Note that persistent large scale structures in the horizontal flow result in time-averaged velocity fields in diagonal pairs of quadrants that are not necessarily identical.

slip velocity boundary conditions in determining the shape and extent of the circulation cells. Such boundary conditions are convenient in allowing one to focus exclusively on the flow that is naturally driven by the plumes, in the absence of the competing, and aspect ratio dependent, effects arising from wall friction. We recognise, however, that practical problems involving confined turbulent plumes necessarily involve wall friction, in addition to the phenomena on which we focus.

For domains of sufficiently large aspect ratio, mass conservation implies that the vertical flux of buoyancy, relative to the ambient buoyancy, $\langle w b\rangle-\langle w\rangle\langle b\rangle / S^{2}$ within a quadrant will be equal to unity in the layer containing the plume and zero in the layer containing the jet. This argument requires the ambient buoyancy in each layer to be uniform, such that the flux of buoyancy, relative to the ambient is zero. Figure $3(c)$ indicates that this is approximately correct for the two largest aspect ratios $S=1,4 / 3$, excepting a relatively small residual flux in the upper layer. In the vicinity of the top boundary at $z=1 / 2$, the relative buoyancy flux $\langle w b\rangle-\langle w\rangle\langle b\rangle / S^{2}$ reduces to zero and the buoyancy in figure $3(a)$ increases, which suggests that the residual buoyancy is transported out of the quadrant horizontally in a steady axisymmetric gravity current.

\subsection{Entrainment}

In general, flows driven by turbulent plumes are interpreted and modelled using plume theory and an entrainment coefficient, under the assumption that each plume acts independently. Entrainment can be estimated from observed volume fluxes or buoyancy differences. In this section we will calculate effective entrainment coefficients $\alpha_{*}$ from observations of volume flux and buoyancy, and use the estimates to identify aspect ratios for which plume theory provides a faithful description of the flow.

In the context of plume theory, the increase in the volume flux in a plume is equal to the rate of entrainment from the surrounding environment. Entrainment is typically parameterised as being proportional to the square root of the momentum flux in the flow, as discussed in Baines \& Turner (1969). The resulting coefficient of proportionality for an isolated unconfined plume is $\alpha \approx 0.12$ (cf. Carazzo et al. 2006. van Reeuwijk \& Craske 2015).

Following Baines \& Turner (1969), there are two ways to calculate an entrainment 
coefficient from the observations reported here. One method is to find the entrainment coefficient from the volume flux at the mid-plane. This could be considered the $a b$ initio estimation of entrainment, because it describes the rate of increase of the volume flux in the plume directly. Alternatively, we can calculate the entrainment coefficient that would be required to predict the observed difference in buoyancy between the upper and lower layers under the assumption that mixing occurs exclusively within the plumes. If the two entrainment rates are equal, they suggest that plume theory provides a faithful description of the flow.

For transparency, we refrain from using a virtual source (Hunt \& Kaye 2001) to account for the finite area of the sources used in the simulations. Indeed, the theoretical location of the virtual source of the infinitely lazy plumes (zero source momentum flux) in this problem coincide with the vertical location of the actual sources, i.e. the theoretical virtual source correction is zero (Hunt \& Kaye 2001). However, figure 2 suggests that, unlike the idealised contraction of lazy plumes that is predicted by plume theory, the near-field behaviour of the plumes in this study is disrupted by background turbulence; hence the use of an effective virtual source might be appropriate. We therefore note that by proceeding without using a virtual source, which would increase $\zeta$ (the distance between the plume source and the interface) in (3.3) and (3.5) below, our estimations of the entrainment coefficient are likely to be slightly larger than they would be if a virtual source were incorporated.

First we will calculate an effective entrainment coefficient from the volume flux at the interface. Inverting $(3.2 a)$ provides a means of estimating the entrainment coefficient from observations of the volume flux at the interface,

$$
\alpha_{Q}=\left(\frac{5}{6}\right)^{3 / 4}\left(\frac{10}{9 \pi^{2}}\right)^{1 / 4} \frac{Q_{m}^{3 / 4}}{\zeta^{5 / 4}} .
$$

where $\zeta$ is the distance from the plume source to the interface and $Q_{m}$ is the volume flux at the level of the interface (plotted in figure 6(a)). The entrainment coefficient estimated from $(3.3)$ is plotted against the aspect ratio $S$ in figure 6 $(c)$ (solid line).

From figure $6(c)$ we see that the entrainment coefficient, $\alpha_{Q}$, calculated from the volume flux at the interface $Q_{m}$, increases with aspect ratio $S$ up to some critical aspect ratio of approximately $S \approx 2 / 3$. Interestingly, this is close to the aspect ratio for which we observed a topological change in the mean flow between quadrants and the emergence of the secondary circulation cells in the upper and lower layers (cf. §3.1). As (3.3) assumes an unstratified ambient, the increase in the estimate of $\alpha_{Q}$ with increasing aspect ratio $S$ at low aspect ratios may be due to the reduction of the stratification in the lower layer as the aspect ratio increases. Increasing the aspect ratio further results in a reduction in the entrainment coefficient, which appears to be tending towards a value that is slightly higher than the value of 0.12 that is commonly associated with an isolated plume in an unconfined environment.

A second method for calculating an effective entrainment coefficient is from the difference in buoyancy between the layers. In a steady state, mass conservation implies that the buoyancy of a given layer is approximately equal to the mean buoyancy $\pm b_{m}$ of the plume supplying fluid to that layer. We define the relative buoyancy flux in each plume as being equal to the volume flux $Q$ multiplied by the buoyancy of the plume relative to the buoyancy of the local environment. The buoyancy flux of a plume just beneath the interface is therefore $Q_{m}\left(b_{m}-\left(-b_{m}\right)\right)=2 Q_{m} b_{m}$. Assuming an unstratified environment in each layer, the buoyancy flux in the plume must be equal to the dimensionless source buoyancy flux, therefore $2 Q_{m} b_{m}=1$, which implies that $b_{m}=1 /\left(2 Q_{m}\right)$. 

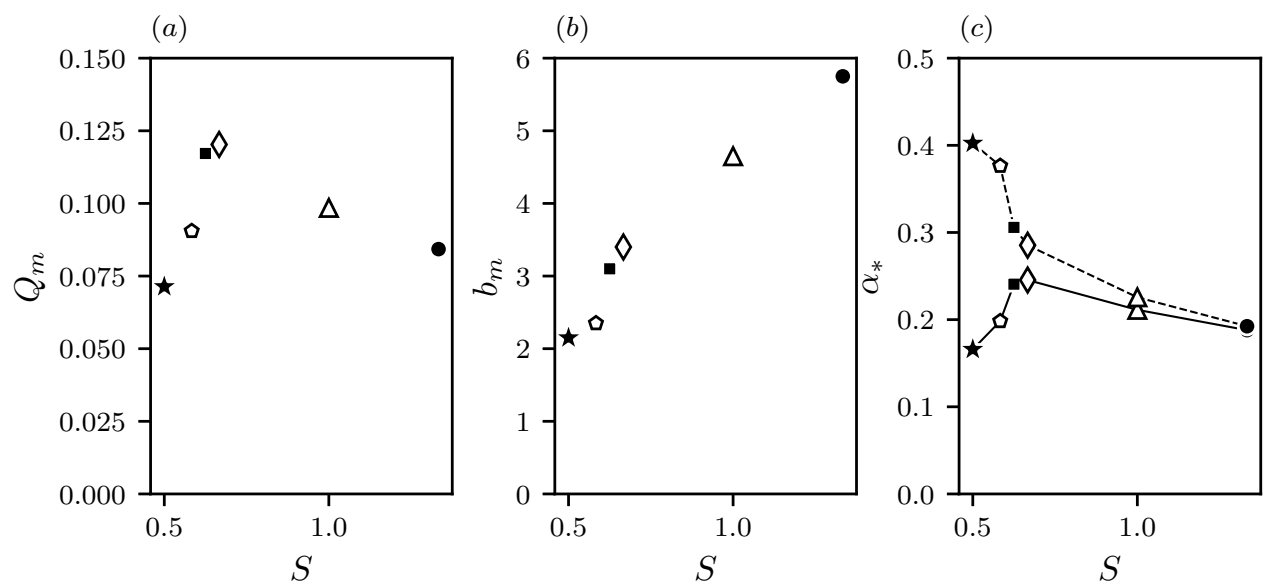

Figure 6. An effective entrainment coefficient using plume theory: $(a)$ The volume flux at the interface $Q_{m},(b)$ half the difference in buoyancy between the layers $b_{m}$, and $(c)$ the effective entrainment coefficient $\alpha_{*}$ for various aspect ratios $S$ (see table 1). An estimate for the entrainment rate $\alpha_{Q}$ (solid line) can be calculated from $Q_{m}$ and equation (3.3). A second estimate for the entrainment coefficient $\alpha_{b}$ (dashed line) is the value required to explain the observed buoyancy difference $b_{m}$ between the layers 3.5 .

Noting that $b_{m}=1 /\left(2 Q_{m}\right)$ and considering $(3.2 a)$, the buoyancy of the upper layer should obey

$$
b_{m}=\frac{5}{12 \alpha}\left(\frac{10}{9 \alpha \pi^{2}}\right)^{1 / 3} \frac{1}{\zeta^{5 / 3}} .
$$

Strictly, the relationship (3.4) between the buoyancy of a layer and the mean buoyancy of the plume by which it is fed is approximate because a small proportion of the plume's total buoyancy flux is provided by turbulent transport, which is formally independent of the mean buoyancy in the plume. In other words, the actual relative buoyancy flux in the plume just beneath the interface is $2 Q_{m} b_{m}$ plus a contribution of approximately $15 \%$ from turbulent transport (van Reeuwijk et al. 2016). Consequently, the buoyancy of a given layer is typically slightly greater in magnitude than the mean buoyancy of the plume might suggest.

We determine $b_{m}$ as half the distance between peaks in the time-averaged probability density function for buoyancy, which is shown in figure 7 and will be discussed in 4.1 . The value of $b_{m}$ for each value of $S$ is plotted in figure $6(b)$. Knowledge of $b_{m}$ and use of equation (3.4) means that the entrainment coefficient can be estimated independently of (3.3) from

$$
\alpha_{b}=\left(\frac{5}{12}\right)^{3 / 4}\left(\frac{10}{9 \pi^{2}}\right)^{1 / 4} \frac{1}{b_{m}^{3 / 4} \zeta^{5 / 4}} .
$$

The entrainment coefficient as calculated from equation (3.5) is plotted in figure 6 $6(c)$ (dashed line). This entrainment rate can be considered as the entrainment rate required as input into a plume model to explain the observed buoyancy difference between the layers.

We observe from figure $6(c)$, that the entrainment coefficient calculated from $b_{m}$, using equation (3.5), results in a value of $\alpha_{b}$ that is everywhere greater than the value of $\alpha \approx 0.12$ that is commonly assigned to isolated and unconfined plumes. Indeed, the use of $\alpha \approx 0.12$ would significantly overestimate the buoyancy difference between the upper 
and lower layers. Figure 6(c) shows that calculating an entrainment coefficient from the buoyancy field using equation (3.5), results in a larger estimate for $\alpha$ than equation (3.3), which is based on the volume flux. The difference can be attributed to two distinct effects. First, the estimation 3.5 implicitly assumes that vertical buoyancy transport can be assigned exclusively to the plumes, rather than interfacial mixing. Secondly, 3.3. does not account for stratification within each layer, which is significant for domains with relatively small aspect ratio and would lead to an increase in the corresponding prediction of $\alpha_{b}$ and a decrease in the predictions of $\alpha_{Q}$.

Figure 6 indicates that for $S=4 / 3$ equations $(3.3)$ and $(3.5)$ provide almost identical and therefore robust estimates for $\alpha$, which suggests that the effects of interfacial mixing are insignificant for $S \geqslant 4 / 3$. However, the estimated value $\alpha_{*} \approx 0.2$ for $S=4 / 3$ is larger than $\alpha \approx 0.12$ for an isolated plume in an unconfined domain. In this regard, the use of an effective virtual source, a distance $\lambda \zeta$ further from the interface than the actual source, would lead to an estimate of $\alpha_{*} \approx 0.2 /(1+\lambda)^{5 / 4}$. However, noting that classical plume theory predicts the virtual source correction for infinitely lazy plumes to be zero (Hunt \& Kaye 2001), we would associate an effective virtual source with the indirect effect that background turbulence has in modifying the near-field development of the plume. In view of the reduction in entrainment with respect to background turbulence reported by (Lai et al. 2019 , Khorsandi et al. 2013 ), attribution of $\alpha_{*}=0.2$ solely to direct effects of background turbulence would therefore be incorrect and misleading, not least because it would also ignore possible modifications to entrainment arising from the mean ambient flow discussed in $\$ 3$.

We conclude by noting that the quadrant aspect ratios $S=1,4 / 3$ approximately correspond to the critical aspect ratio $H / R=1$ for overturning identified by Baines \& Turner (1969), if the radius $R$ of the cylinder containing their single plume is compared with the shortest distance $S H$ between the plume axes of the present problem.

\section{Flow energetics}

Unlike the volume flux $Q_{m}$ and the upper and lower layer buoyancy $\pm b_{m}$, there are quantities that can be predicted directly from the system's energy budget. An example is the total viscous dissipation, which is necessarily equal to the volumetric integral of the buoyancy flux, regardless of the strength of the circulations and buoyancy differences studied in the previous section. In the following section, we utilise such properties to derive models for the flow's energetics that do not involve an entrainment coefficient.

We will start by describing the energetics framework, defining the local available potential energy and background potential energy (BPE) in 4.1 . We then discuss the viscous dissipation and BPE production in $\$ 4.2$, before describing models in $\$ 4.3+84.5$. In $\$ 4.6$ and 4.7 we examine the similarity arguments upon which these models are based.

\subsection{Governing equations for energetics}

Let $z_{*}(b, t)$ represent an adiabatic rearrangement of the fluid into the configuration possessing the minimal potential energy and, therefore, a monotonically increasing reference buoyancy $b_{*}(z, t)$. The minimal potential energy is equal to the BPE (Lorenz 1955). For this problem, in which $z_{*} \in[-0.5,0.5]$, the quantity $\partial z_{*} / \partial b$ corresponds to the probability density function for buoyancy. Indeed, $z_{*}$ can be obtained during a simulation by integrating the probability density function for buoyancy (Winters et al. 1995). The histograms of the time average of $\partial z_{*} / \partial b$ for different aspect ratios shown in figure 7 demonstrate that as the domain aspect ratio increases the variance of the global distribution of buoyancy increases. 


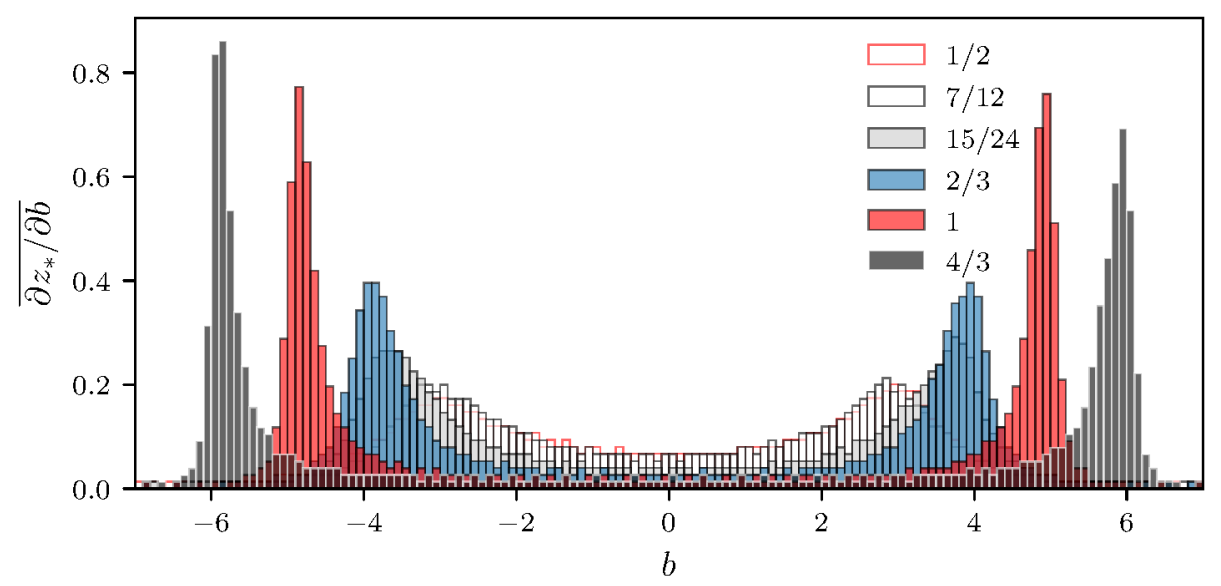

FIGURE 7 . The time-averaged probability density function of the buoyancy, which corresponds to $\partial z_{*} / \partial b$, over the entire domain for different aspect ratios.

When non-dimensionalised, the local gravitational potential energy of the fluid is $E_{p} \equiv$ $-b z$. Typically, the portion of the potential energy that is available to do work to increase the kinetic energy of the flow is computed as the volume integral of $b\left(z_{*}-z\right)$ (Winters et al. 1995). Locally, however, $b\left(z_{*}-z\right)$ is not positive definite, which motivates the definition of a suitable local APE density. Following Holliday \& Mcintyre (1981) and Scotti \& White (2014), we therefore consider the positive semidefinite quantity

$$
E_{a} \equiv \int_{b_{*}}^{b}\left(z_{*}(\hat{b}, t)-z\right) \mathrm{d} \hat{b} .
$$

Physically, the definition (4.1) is positive semidefinite because it accounts for both the potential energy associated with a parcel of fluid displaced from equilibrium and the potential energy associated with the corresponding displacement of the environment, as can be seen by decomposing (4.1),

$$
E_{a}=b\left(z_{*}-z\right)-\int_{0}^{z-z_{*}} b_{*}(z-\hat{z}, t) \mathrm{d} \hat{z},
$$

where $b_{*}(z, t)$ is the reference buoyancy, such that $z_{*}\left(b_{*}(z, t), t\right)=z$, corresponding to the adiabatically rearranged state possessing minimal potential energy. The quantity $E_{a}$ therefore has a dependence on the structure of the entire buoyancy field, i.e. the value of $E_{a}$ at a point may change because parcels of fluid mixing at locations that are remote from that point leads to a modification of $b_{*}$. In the problem that we consider the reference buoyancy can be equated with the ambient buoyancy $\pm b_{m}$, except over thin layers at the bottom, middle and top of the domain. Using (2.3), the Lagrangian derivative of $E_{a}$ satisfies (Scotti \& White 2014)

$$
\frac{D E_{a}}{D t}=\frac{\nabla^{2} E_{a}}{P e}+\frac{2}{P e} \frac{\partial\left(b-b_{*}\right)}{\partial z}-G-w\left(b-b_{*}\right)+\int_{b_{*}}^{b} w_{*}(\hat{b}, t) \mathrm{d} \hat{b},
$$


where the APE dissipation

$$
G \equiv \frac{1}{P e}\left(\left.|\nabla b|^{2} \frac{\partial z_{*}}{\partial b}\right|_{b}-\left.\left|\nabla b_{*}\right|^{2} \frac{\partial z_{*}}{\partial b}\right|_{b_{*}}\right), \quad \text { and } \quad w_{*} \equiv \frac{\partial z_{*}}{\partial t} .
$$

The final term in 4.3 is zero when the probability density function for buoyancy (cf. figure 7) does not depend on time, which is true in the present context for domains that are of sufficiently large aspect ratio. In that case, equation 4.3 reduces to

$$
\frac{\partial E_{a}}{\partial t}+\nabla \cdot\left(\boldsymbol{u} E_{a}\right)=\frac{\nabla^{2} E_{a}}{P e}+\frac{2}{P e} \frac{\partial\left(b-b_{*}\right)}{\partial z}-G-\underbrace{w\left(b-b_{*}\right)}_{-\Phi_{z}},
$$

in which $-\Phi_{z}$ is a buoyancy flux relative to the reference state.

Following Scotti \& White (2014), we define the local BPE density $E_{b}$ as $E_{b} \equiv-b z-E_{a}$, whose budget obeys

$$
\frac{\partial E_{b}}{\partial t}+\nabla \cdot\left(\boldsymbol{u} E_{b}\right)=\frac{\nabla^{2} \psi}{P e}-\nabla \cdot\left(\boldsymbol{u} p_{*}\right)+\underbrace{\left.\frac{|\nabla b|^{2}}{P e} \frac{\partial z_{*}}{\partial b}\right|_{b}}_{\Phi_{d}},
$$

where

$$
\psi=-\int_{0}^{b} z_{*}(\hat{b}) \mathrm{d} \hat{b}, \quad p_{*}=\int_{0}^{z} b_{*}(\hat{z}) \mathrm{d} \hat{z} .
$$

In a Boussinesq flow, the mixing rate $\Phi_{d}$ represents an irreversible conversion of APE into BPE via diapycnal mixing. An interesting feature of $\Phi_{d}$, which proves to be crucial when considering energetics and entrainment, is that it corresponds to the dissipation of buoyancy variance $|\nabla b|^{2} / P e$ weighted by the probability density function $\partial_{b} z_{*}$. Diapycnal mixing therefore has more energetic significance when it accounts for mixing in regions of buoyancy whose probability density is relatively large.

In the subsequent analysis, we choose to focus on the production of $\operatorname{BPE} \Phi_{d}$ rather than the APE dissipation $G$, because it is the volume integral of $\Phi_{d}$ that features explicitly in previous work on bulk energetics (Winters et al. 1995, Hughes et al. 2013 , for example) and evaluates to unity in this particular problem (see equation (4.11)). We note that locally BPE production, $\Phi_{d}$, is not necessarily equal to APE dissipation $G$, because the latter only accounts for mixing resulting from macroscopic motion in the flow and not the mixing $\left(\partial z_{*} / \partial b\right)\left|\nabla b_{*}\right|^{2} / P e$ associated with diffusion of the background state; hence $\Phi_{d} \geqslant G$ for the Boussinesq model considered here. However, in the present problem, the rearranged state consists primarily of two constant density layers in which $\nabla b_{*} \approx 0$ (see, for example, figure 7). With the possible exception of thin layers near the horizontal boundaries, $\Phi_{d}$ and $G$ are therefore approximately equal, because they are dominated by the large gradients of $b$ within the plumes. In regarding local BPE production as equivalent to APE dissipation for this particular Boussinesq flow we are not endorsing the use of BPE production to characterise mixing more generally. The energy conversion implied by BPE production is different to APE dissipation (Tailleux 2009) and in nonBoussinesq models, for which BPE 'production' can be negative (Tailleux 2009, Gregg et al. 2018), the difference is significant.

The BPE production over vertical and horizontal slices is displayed in figures 8 and 9. respectively. These figures illustrate that regions of high $\Phi_{d}$ typically occur on thin surfaces at the instantaneous edge of the plumes (cf. figure 2 corresponding to the same 


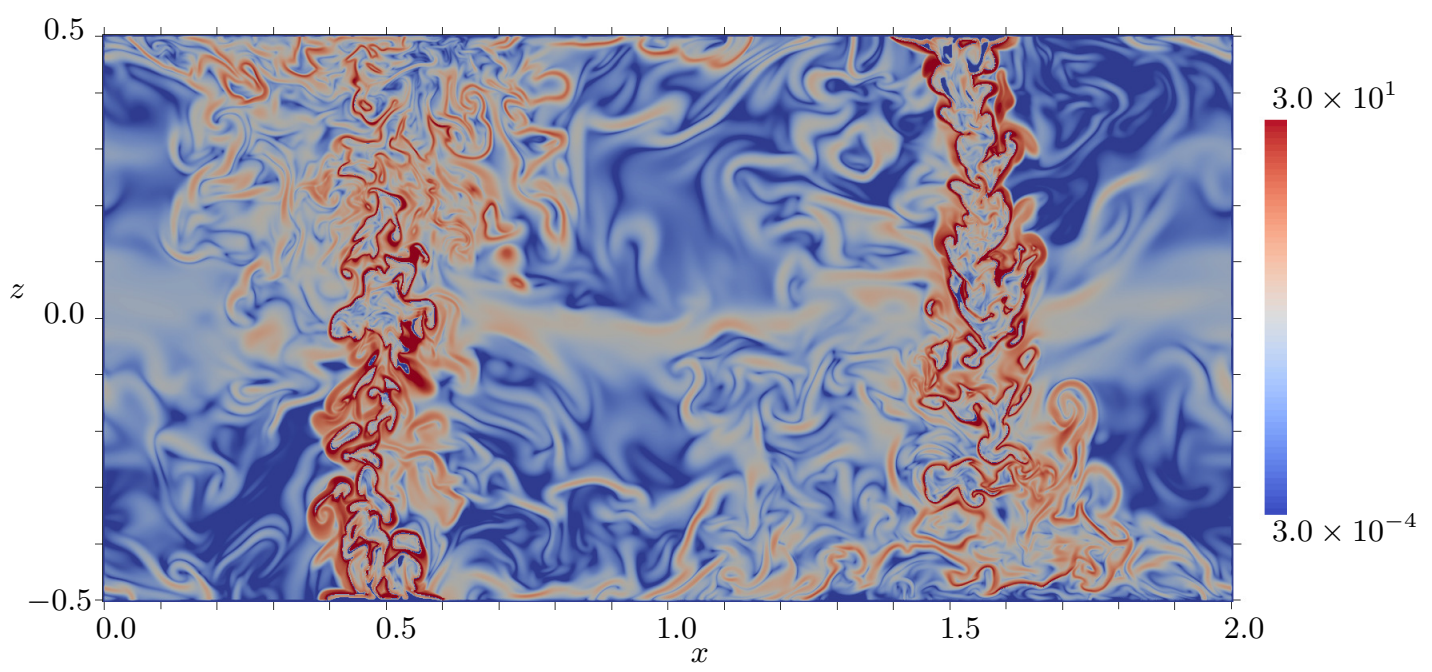

Figure 8 . The BPE production $\Phi_{d}=\left(\mathrm{d} z_{*} / \mathrm{d} b\right)|\nabla b|^{2} / P e$ over a vertical slice of the domain using a logarithmic scale. The domain has a quadrant aspect ratio of $S=1$ and the slice intersects the vertical axis of two of the plumes. The slice was taken at the same time as that of the buoyancy field displayed in figure 2

point in time), where both $|\nabla b|$ and the probability density $\partial z_{*} / \partial b$ are relatively large because the buoyancy is close to a background buoyancy $\pm b_{m}$. Diapycnal mixing is energetically insignificant in the vicinity of the interface outside the plumes for the aspect ratio $S=1$ shown in figure 8 , although the instantaneous picture of figure 8 does not necessarily account for intermittent events that would increase the mean BPE production.

The local kinetic energy density $E_{k}$ (per unit mass) is defined according to $E_{k} \equiv|\boldsymbol{u}|^{2} / 2$, and satisfies

$$
\frac{\partial E_{k}}{\partial t}+\nabla \cdot\left(\boldsymbol{u} E_{k}\right)=-\nabla \cdot\left(\boldsymbol{u}\left(p-p_{*}\right)\right)+\underbrace{w\left(b-b_{*}\right)}_{-\Phi_{z}}+\frac{1}{R e} \nabla^{2} E_{k}-\varepsilon .
$$

We will refer to the term $\varepsilon \equiv\left(\partial_{j} u_{i}\right)^{2} / R e$ as the viscous dissipation, noting that, prior to spatial integration, the time average of $\varepsilon$ is strictly only equivalent to the true viscous dissipation at a given point in homogeneous turbulence. The physical role played by the relative buoyancy flux $-\Phi_{z}$ in 4.5 and 4.8 is the reversible conversion of APE into kinetic energy (Winters et al. 1995).

The viscous dissipation $\varepsilon$ of kinetic energy in (4.8) depends exclusively on local quantities, in the sense that it is evaluated from properties of the velocity field at a single point. In contrast, the BPE production $\Phi_{d}$ (and APE dissipation) depends explicitly on the global probability density function for buoyancy. As illustrated in figure 10 , $\varepsilon$ is relatively large in the core of the plume, which is enveloped by surfaces on which $\Phi_{d}$ is maximised. Such surfaces correspond to relatively large values of both $|\nabla b|$ and the probability density function for buoyancy (i.e. surfaces on which $b \approx \pm b_{m}$, corresponding approximately to the the white lines in figure 2.

\subsection{Integrals and statistics for energetics}

The quadrant volume integrals of $\Phi_{z}, \varepsilon$ and $\Phi_{d}$, multiplied by a factor of 2 , are displayed in table 2. The factor of 2 was introduced to ensure that the theoretical predictions of $\Phi_{z}$, 

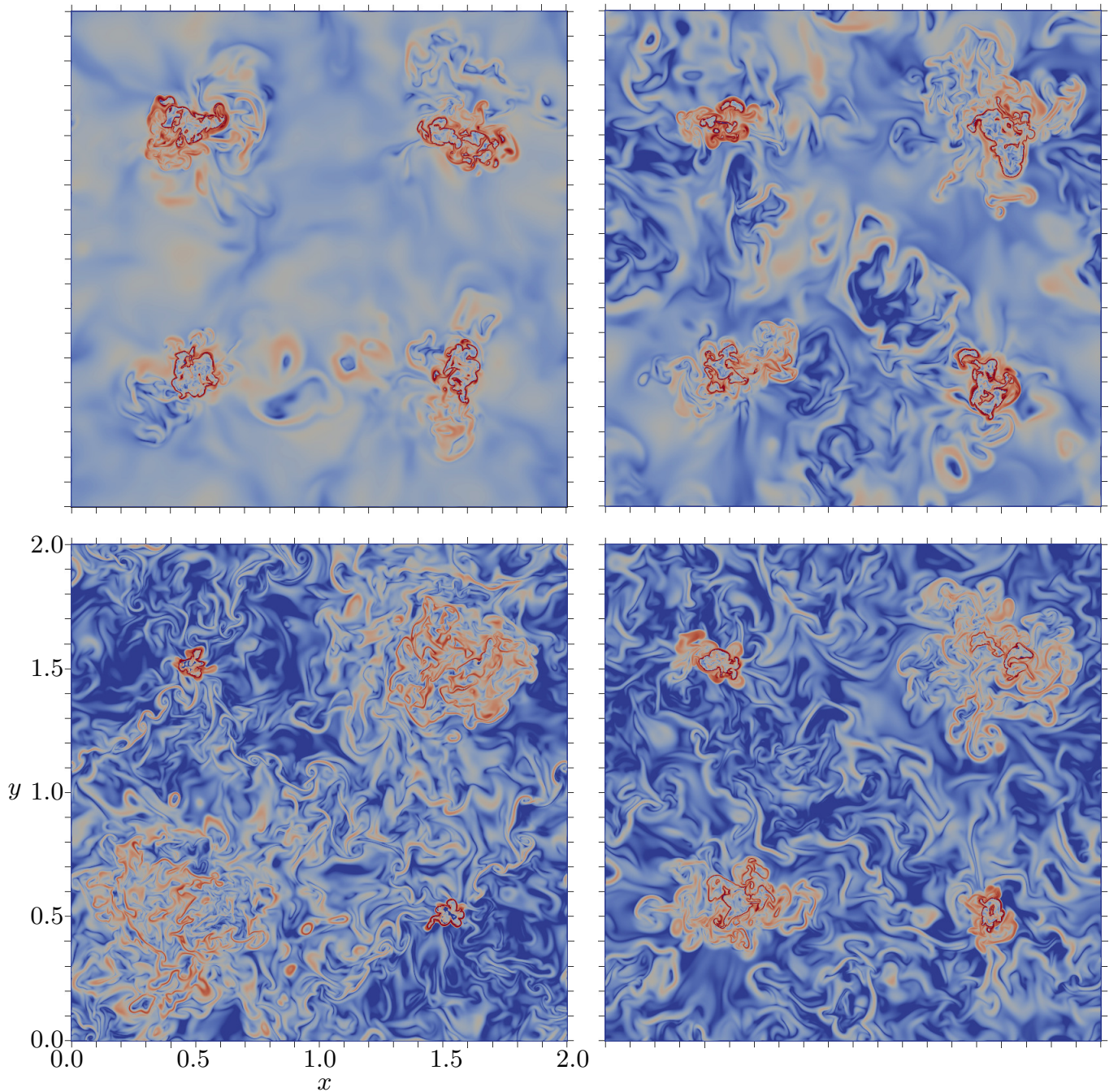

Figure 9. The BPE production $\Phi_{d}=\left(\mathrm{d} z_{*} / \mathrm{d} b\right)|\nabla b|^{2} / P e$ over horizontal slices of the domain using a logarithmic scale. The slices were taken at $z=0,0.1,0.2,0.45$ clockwise from top left. The plumes in each slice can be seen in the top-left and bottom-right quadrants of each window and the jets in the bottom-left and top-right quadrants. For the colour scale used in the figure see figure 8 .

$\varepsilon$ and $\Phi_{d}$ each correspond to unity. The values indicate that, whilst substantially more BPE production takes place in the lower layer than in the upper layer, the distribution of viscous dissipation in each of the quadrant's layers is approximately uniform.

Integration of 4.8 over the volume of a quadrant and time, indicates that the integral of viscous dissipation is equal to the work done by buoyancy,

$$
\langle\langle\varepsilon\rangle\rangle=-\left\langle\left\langle\Phi_{z}\right\rangle\right\rangle
$$

where $\left\langle\left\langle\Phi_{z}\right\rangle\right\rangle=-\left\langle\left\langle w\left(b-b_{*}\right)\right\rangle\right\rangle=-\langle\langle w b\rangle\rangle$, because $b_{*}$ does not depend on $x$ or $y$ and therefore $\left\langle\left\langle w b_{*}\right\rangle\right\rangle$ is identically zero. It is interesting that for the two largest aspect ratios $\left\langle\left\langle\Phi_{z}\right\rangle\right\rangle$ is greater than 1 , which exceeds the buoyancy flux provided by the sources. The 


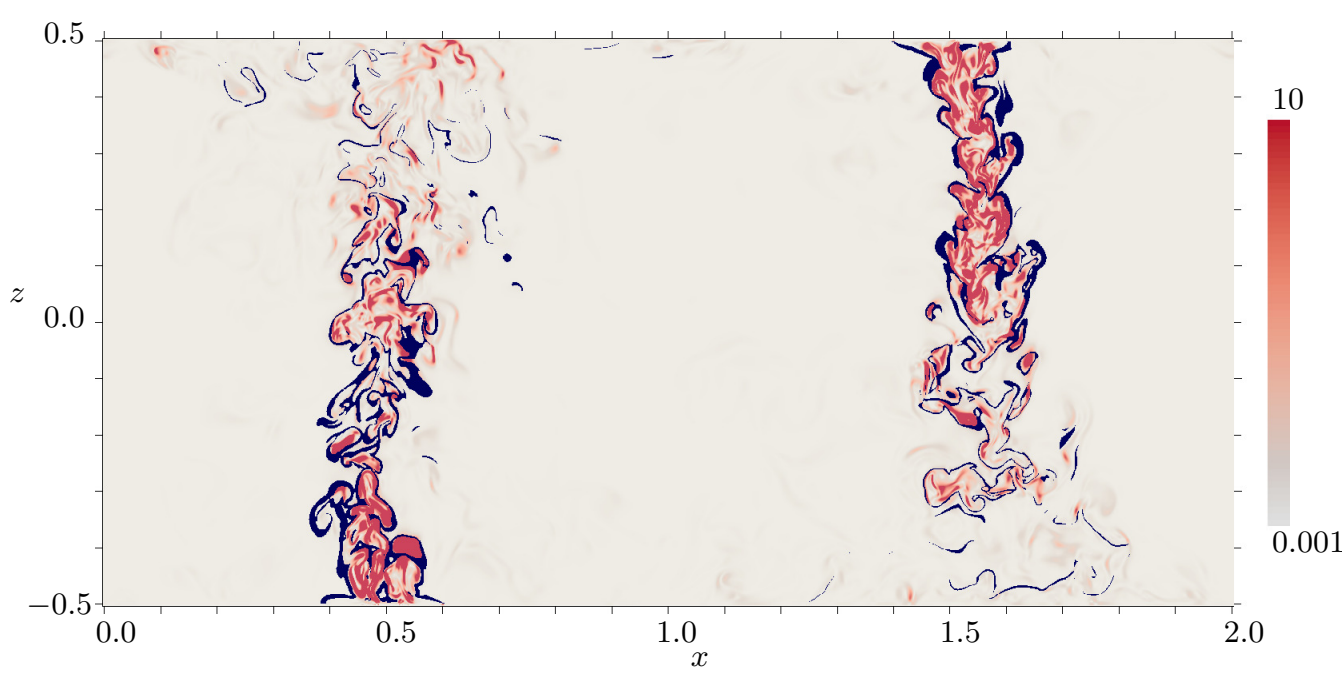

FIGURE 10. Regions of viscous dissipation $\varepsilon=\left(\partial_{j} u_{i}\right)^{2} /$ Re coloured using a logarithmic scale. The thin dark (blue) parts of the figure denotes regions in which the BPE production $\Phi_{d}=\mathrm{d} z_{*} / \mathrm{d} b|\nabla b|^{2} / P e \geqslant 3$.

cause of the disparity is seen when the governing equation for buoyancy (2.3) is multiplied by $z$ to give a volumetric budget for the potential energy $E_{a}+E_{b}=-b z$, which implies that

$$
-\left\langle\left\langle\Phi_{z}\right\rangle\right\rangle-\left\langle\left\langle\Phi_{i}\right\rangle\right\rangle=\underbrace{-\frac{1}{P e}\left\langle\frac{\partial b}{\partial z} z\right\rangle_{-\zeta}^{\zeta}}_{=1 / 2},
$$

over a single quadrant. The right-hand side of 4.10 is equal to $1 / 2$ because a given quadrant contains a single source of buoyancy at either $z=-1 / 2$ or $z=1 / 2$. The input of potential energy at the boundaries is therefore equal to the sum of the integral of the convective buoyancy flux and diffusion of buoyancy down its mean profile $-\left\langle\left\langle\Phi_{i}\right\rangle\right\rangle \equiv$ $-\left\langle\left\langle P e^{-1} \partial_{z} b\right\rangle\right\rangle$. Indeed, there is a flux of buoyancy due to the plume in addition to a (negative) diffusive flux down the stable background stratification. Together, these contributions result in $\left\langle\left\langle\Phi_{z}\right\rangle\right\rangle$ being either slightly higher or slightly lower than the input at the boundaries, depending on the area of the plume sources, relative to the area of the horizontal boundaries. Nevertheless, the contribution from $\left\langle\left\langle\Phi_{i}\right\rangle\right\rangle$ is small relative to the buoyancy flux $\left\langle\left\langle\Phi_{z}\right\rangle\right\rangle$, due to the relatively high Péclet number of the flow, which results in a close agreement between $-\left\langle\left\langle\Phi_{z}\right\rangle\right\rangle$ and the surface buoyancy fluxes in 4.10 .

Unlike the viscous dissipation, which relies on the conversion of APE to kinetic energy via $\left\langle\left\langle\Phi_{z}\right\rangle\right\rangle$, the volumetric production of BPE can be calculated directly from surface fluxes,

$$
\left\langle\left\langle\Phi_{d}\right\rangle\right\rangle=\underbrace{\frac{1}{P e}\left\langle\frac{\partial b}{\partial z} z_{*}\right\rangle_{-\zeta}^{\zeta}}_{=1 / 2},
$$

for sufficiently long time averages, as is verified to within approximately $1 \%$ by the values (scaled by a factor of 2 ) in table 2 . The data in table 2 also show that the total input of APE at the boundaries, which is equal to twice the input of potential energy, 


$\begin{array}{cccccccccc}S & -\left\langle\left\langle\Phi_{z}\right\rangle\right\rangle & -\left\langle\left\langle\Phi_{z}\right\rangle\right\rangle_{-\zeta}^{0} & -\left\langle\left\langle\Phi_{z}\right\rangle\right\rangle_{0}^{\zeta} & \langle\langle\varepsilon\rangle\rangle & \langle\langle\varepsilon\rangle\rangle_{-\zeta}^{0} & \langle\langle\varepsilon\rangle\rangle_{0}^{\zeta} & \left\langle\left\langle\Phi_{d}\right\rangle\right\rangle & \left\langle\left\langle\Phi_{d}\right\rangle\right\rangle_{-\zeta}^{0} & \left\langle\left\langle\Phi_{d}\right\rangle\right\rangle_{0}^{\zeta} \\ 0.50 & 0.991 & 0.827 & 0.164 & 0.979 & 0.584 & 0.395 & 1.008 & 0.781 & 0.227 \\ 0.58 & 1.006 & 0.839 & 0.167 & 1.020 & 0.593 & 0.428 & 1.005 & 0.780 & 0.225 \\ 0.62 & 0.982 & 0.870 & 0.111 & 0.981 & 0.574 & 0.407 & 1.014 & 0.801 & 0.213 \\ 0.67 & 0.992 & 0.899 & 0.094 & 0.990 & 0.535 & 0.455 & 1.003 & 0.758 & 0.244 \\ 1.00 & 1.003 & 0.918 & 0.085 & 1.005 & 0.492 & 0.512 & 0.997 & 0.738 & 0.259 \\ 1.33 & 1.005 & 0.923 & 0.082 & 1.004 & 0.459 & 0.545 & 0.998 & 0.751 & 0.247 \\ & & & & & & & & & \\ \text { Theory } & 1.000 & 1.000 & 0.000 & 1.000 & 0.500 & 0.500 & 1.000 & 0.750 & 0.250\end{array}$

TABLE 2. Domain decomposition of the buoyancy flux $\left\langle\left\langle\Phi_{z}\right\rangle\right\rangle$, viscous dissipation $\langle\langle\varepsilon\rangle\rangle$ and BPE production $\left\langle\left\langle\Phi_{d}\right\rangle\right\rangle \cdot\langle\langle\rangle\rangle,\langle\langle\rangle\rangle_{-\zeta}^{0}$ and $\langle\langle\rangle\rangle_{0}^{\zeta}$ refer to volume integrals over an entire quadrant, the (lower) plume layer and the (upper) jet layer, respectively. The row labelled 'Theory' corresponds to the results obtained for Gaussian profiles in 4.3 and $\$ 4.4$. Noting that each layer of the domain has height 0.5 , the volume integrals displayed in this table have been multiplied by a factor of 2 , to facilitate a comparison with the horizontal axis of figure 11 and the interpretation of their ratios.

is split equally between BPE production and viscous dissipation, leading to the mixing efficiency of approximately $1 / 2$ that is also found in Rayleigh-Bénard convection (Hughes et al. 2013).

Table 2 indicates that the viscous dissipation in the lower layer $\langle\langle\varepsilon\rangle\rangle_{-\zeta}^{0}$ generally decreases with increasing aspect ratio. In general, and particularly in domains of small aspect ratio, turbulence is transported horizontally out of a quadrant's upper layer (which, for the purpose of discussion, we assume contains the jet) and into neighbouring quadrants. At larger aspect ratios a greater proportion of this turbulence is dissipated before it is transported out of the upper layer, leading to the observed decrease in lowerlayer viscous dissipation for large aspect ratios.

Figure 11 displays horizontal integrals of $(a)$ the BPE production $\left\langle\Phi_{d}\right\rangle$ and $(b)$ the viscous dissipation $\langle\varepsilon\rangle$ over the quadrant as a function of $z$. Consistent with the information in table 2, figure 11 indicates that the BPE production is significantly higher in the plume below the interface than it is in the jet above the interface, in which it decays with respect to $z$. In the lower layer the BPE production exhibits a local maximum of approximately 0.8 for the largest aspect ratio. Due to the finite area of the source, the production of BPE production increases significantly in the vicinity of the bottom boundary. In the following sections we develop the integral model whose predictions are compared to the observations in figure 11 .

\subsection{Viscous dissipation}

In this section we will develop a bulk model for the volume integral of viscous dissipation $\langle\langle\varepsilon\rangle$ in the quadrant layer that contains a jet and the quadrant layer that contains a plume. We will also describe a model for the vertical variation of horizontally integrated viscous dissipation in each layer.

For generality we will assume that the distance from the plume source to the interface is $0 \leqslant \zeta \leqslant 1$, and not necessarily equal to $1 / 2$. In practice, the situation we have in mind might correspond to an 'emptying filling box' model of a space ventilated by low and high-level openings (Linden et al. 1990) or to a domain containing an unequal number of buoyancy sources on its bottom boundary compared with its top boundary. 
(a)

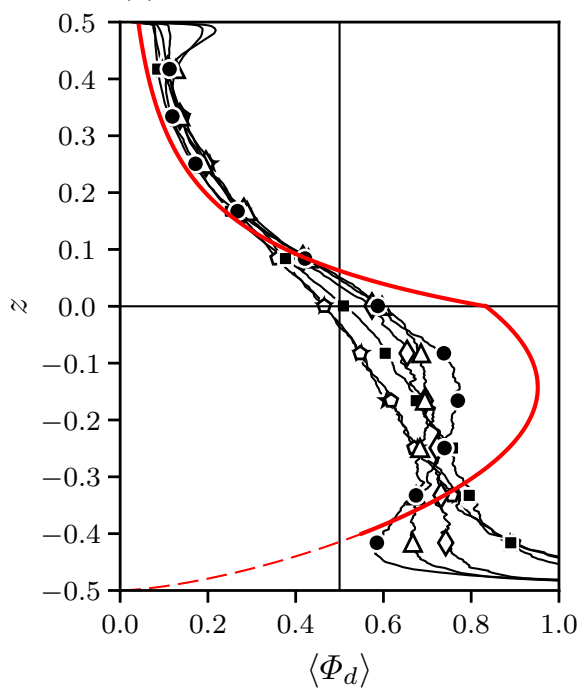

(b)

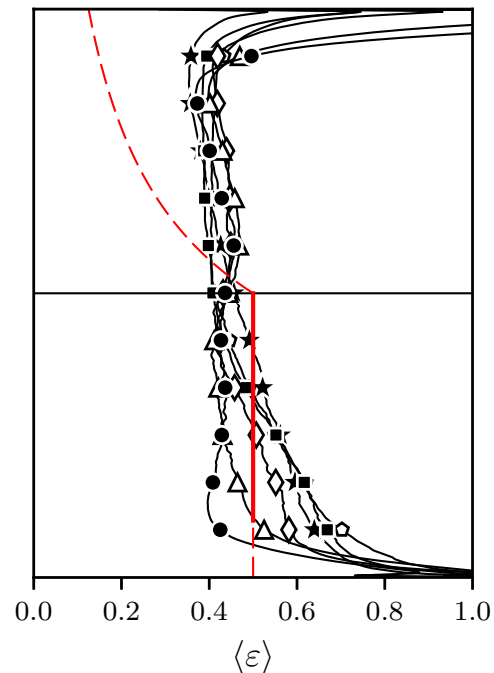

Figure 11. (a) BPE production and (b) viscous dissipation over a quadrant of the domain. In this quadrant the plume occupies the lower layer and the jet occupies the upper layer. The thick (red) lines correspond to the theoretical predictions. The model in $(a)$ is developed in 84.5 . The model is dashed in regions close to the source, because it was developed under the assumption of point sources of buoyancy flux. The thick line in $(b)$ is the total dissipation in the lower layer (4.13), divided by the layer height, which gives $\langle\varepsilon\rangle=1 / 2$ for a Gaussian velocity profile. The dashed line in $(b)$ is described by equation (4.14) and corresponds to viscous dissipation in an unconfined jet. For the symbols used to denote each aspect ratio see table 1 .

For consistency with the previous sections, we continue to regard the ambient buoyancy of the upper layer as $b_{m}$ and that of the lower layer as $-b_{m}$.

In the absence of a direct input of kinetic energy at the boundaries, the viscous dissipation in the domain is balanced by the total work undertaken by buoyancy. Buoyancy is only able to do work on the flow in the quadrant's lower layer $(z<0)$, because in the upper layer the mean buoyancy difference between the plume and the ambient is zero. As discussed in 84.2 provided that the vertical buoyancy transport by diffusion is small compared with the relative buoyancy flux $w\left(b-b_{*}\right)$, the kinetic energy dissipation is equal to the buoyancy flux multiplied by $\zeta$, which is the dimensionless distance over which buoyancy is able to do work in a single quadrant.

Assuming that the aspect ratio is sufficiently large and that the upper layer is not stratified, all the kinetic energy that is transported across the interface is dissipated in the upper layer. In other words, the total kinetic energy dissipation in the layer containing the jet is equal to the transport of kinetic energy across the interface.

Using the steady-state plume solutions 3.2 , and noting that all quantities are nondimensionalised using the source buoyancy flux and domain height, the transport of kinetic energy across the interface at $z=0$ is

$$
\left.\left\langle\frac{w^{3}}{2}\right\rangle\right|_{z=0}=\frac{\gamma M_{m}^{2}}{2 Q_{m}}=\frac{3 \gamma}{8} \zeta,
$$

where $\gamma$ is therefore a parameter that accounts for the shape of the velocity profile and turbulent transport (see, e.g. Priestley \& Ball 1955; van Reeuwijk \& Craske 2015). Note that equation 4.12 relates to the kinetic energy budget for which the forcing from the 
sources of buoyancy is known, and therefore does not involve the entrainment coefficient $\alpha$. For the same reason equation 4.12 , unlike the volume flux $Q$, would not be directly affected by the division of a plume into multiple plumes that provide the same combined buoyancy flux.

The total kinetic energy dissipation in the layer containing the plume is equal to the integral of the buoyancy flux over the layer, minus the transport of kinetic energy between the top and bottom layers; hence

$$
\langle\langle\varepsilon\rangle\rangle_{-\zeta}^{0} \equiv \int_{-\zeta}^{0}\langle\varepsilon\rangle \mathrm{d} z=\zeta\left(1-\frac{3 \gamma}{8}\right) .
$$

This result can also be obtained from the plume equations by integrating the production term in the integral energy transport equation (see, e.g. van Reeuwijk \& Craske 2015). Equations (4.12) and (4.13) state that when $\gamma=4 / 3$, which corresponds to a Gaussian velocity profile (van Reeuwijk \& Craske 2015), there is equal viscous dissipation in the upper layer compared with the lower layer.

In the plume, self-similarity implies that the approximately constant integral buoyancy force doing work on the flow results in the horizontal integral of viscous dissipation being constant. The viscous dissipation in the plume for $z \leqslant 0$ is therefore equal to $1-3 \gamma / 8=1 / 2$ for a Gaussian velocity profile, as indicated by the red solid line in the lower half of figure $11(b)$. In contrast, fluid in a jet is not subjected to a mean force from buoyancy, which results in its viscous dissipation decaying with respect to $z$. Equating the jet's dissipation with the vertical derivative of its energy flux $\gamma M^{2} / 2 Q$ using (3.1) implies that

$$
\langle\varepsilon\rangle=\frac{3 \gamma}{8} \frac{\zeta^{2}}{(\zeta+z)^{2}}, \quad z \geqslant 0 .
$$

We include (4.14) as a dashed curve in the upper layer of figure $11(b)$ as a reference to the behaviour of an unconfined jet. We note, however, that (4.14) does not give accurate predictions in the vicinity of the top boundary due to vertical confinement. Indeed, the value $2\langle\langle\varepsilon\rangle\rangle_{0}^{\zeta}=0.5$ in table 2 can only be obtained by integrating 4.14 from $z=0$ to $\infty$.

\subsection{An integral model for the production of BPE}

In this section we will develop a model for the integral production of BPE in the layer containing the jet and the layer containing the plume. As discussed in 4.1 . our model does not distinguish between BPE production $\Phi_{d}$ and APE dissipation $G$, which readers can regard as interchangeable in the following model, in spite of the significant differences between $\Phi_{d}$ and $G$ in more general cases (see, for example, Tailleux 2009). In a stable two-layer stratification consisting of an upper and lower layer of buoyancy $b_{m}$ and $-b_{m}$, respectively, the sorted elevation of fluid parcels is

$$
z_{*}(b)=\left\{\begin{array}{lc}
1-\zeta, & b_{m}<b, \\
0, & -b_{m}<b<b_{m}, \\
-\zeta, & b<-b_{m} .
\end{array}\right.
$$

Parcels whose buoyancy is exactly equal to $-b_{m}$ and $b_{m}$ occupy the regions $-\zeta<z_{*}<0$ and $0<z_{*}<1-\zeta$, respectively. Using 4.2 , the local APE can be evaluated as 


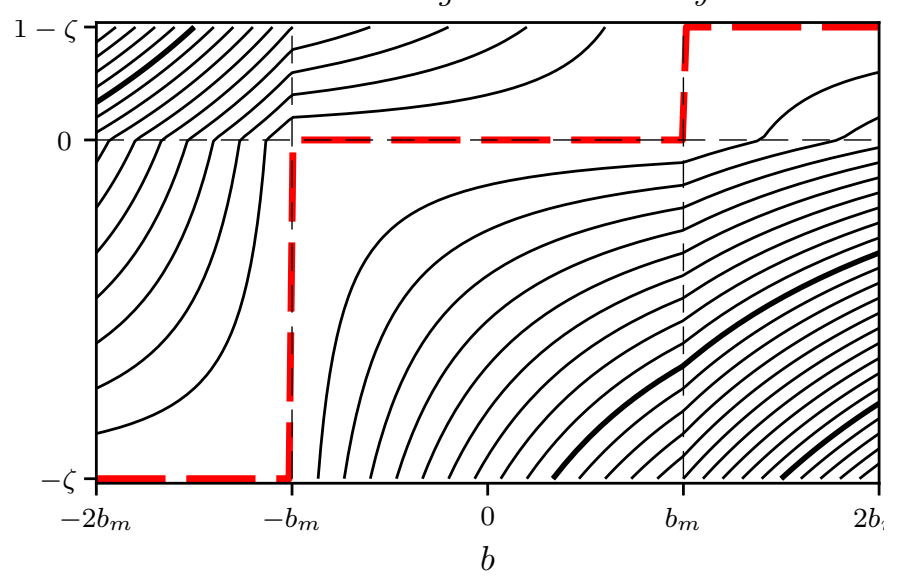

Figure 12. Contours of the local available potential energy $E_{a}$ according to 4.16 , with respect to the buoyancy and vertical position for parcels displaced from a stable two-layer stratification. The thick dashed line corresponds to $z_{*}$, which minimises $E_{a}$. The spacing of the contours is equal to 0.1 .

$$
E_{a}(b, z)=b\left(z_{*}-z\right)+b_{m}\left(|z|-\left|z_{*}\right|\right),
$$

whose contours are displayed in figure 12. Note that $E_{a}\left(b_{m}, z\right)=0$ for $z>0$ and $E_{a}\left(-b_{m}, z\right)=0$ for $z<0$. Conceptually 4.16 states that APE comes either from negatively buoyant parcels of fluid displaced upwards from $z<0$ or from positively buoyant parcels of fluid displaced downwards from $z>0$, as illustrated either side of the interface in figure 13. For arbitrary interface heights $\zeta, E_{a}$ is not symmetric around the interface, because the vertical distance to equilibrium for parcels of fluid with $b<-b_{m}$ is not necessarily the same as it is for parcels with $b>b_{m}$.

Multiplication of equation 4.16 by $w$ and integration over a horizontal slice (i.e. application of $\langle\cdot\rangle)$ gives an expression for the flux of APE in the jet. Noting that $\left\langle\Phi_{z}\right\rangle=$ $-\left\langle w\left(b-b_{*}\right)\right\rangle=0$ when $z>0$ (because in the upper layer the jet has the same mean buoyancy as the ambient), the flux can be expressed as

where

$$
\left\langle w E_{a}\right\rangle=\frac{1-\zeta}{2}\left\langle\left|\Phi_{z}\right|\right\rangle
$$

$$
\frac{\left\langle\left|\Phi_{z}\right|\right\rangle}{2}=\int_{b>b_{m}} w\left(b-b_{m}\right) \mathrm{d} x \mathrm{dy},
$$

corresponds to the quadrant integral of the flux $\Phi_{z}$ over regions where $b<b_{m}$.

We proceed by assuming that for domains of sufficiently large aspect ratio, the ambient is well-mixed and of uniform buoyancy and, therefore, that the horizontal flux of APE through the sides of the quadrant is equal to zero. Our observations of the mean horizontal flux of APE out of a quadrant indicate that it is negligible for domains with aspect ratio $S \geqslant 1$. If there is no transport of APE out of a quadrant, the APE flux $\left\langle w E_{a}\right\rangle$ at the the level of the interface is equal to the total dissipation of APE in the upper layer. Note that in the upper layer the buoyancy flux $-\left\langle\Phi_{z}\right\rangle$ is zero; hence the overall conversion of APE to kinetic energy is also zero: 


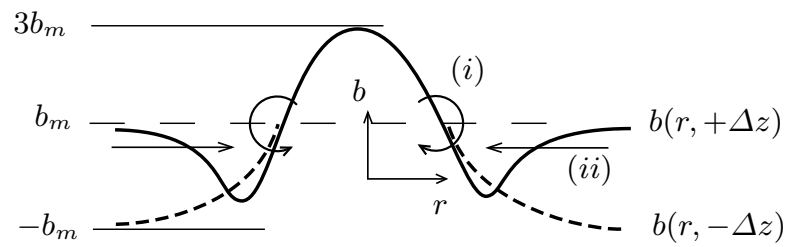

Figure 13. Schematic description of the mean buoyancy profile a small distance above $(\Delta z)$ and below $(-\Delta z)$ the interface. When the plume crosses the interface between the two layers it moves from a layer with mean buoyancy $-b_{m}$ to a layer with mean buoyancy $b_{m}$. The buoyancy profile changes from $b(r,-\Delta z)$ (thick dashed line) to $b(r,+\Delta z)$ (thick solid line). The core of the jet is positively buoyant relative to the layer buoyancy, shown by the central part of the solid line being above $b_{m}$, while the outer shell of the plume is negatively buoyant relative to the layer buoyancy. As the jet travels through the layer, the radial buoyancy variation in the jet is mixed out. We model this as (i) mixing between the core and the outer shell and (ii) entrainment from outside the jet into the outer shell. In 4.7 we quantify the role of turbulent fluctuations that modify this simple heuristic picture.

$$
\langle\langle G\rangle\rangle_{0}^{1-\zeta} \approx\left\langle\left\langle\Phi_{d}\right\rangle_{0}^{1-\zeta}=\int_{0}^{1-\zeta}\left\langle\Phi_{d}\right\rangle \mathrm{d} z=(1-\zeta) \beta\right.
$$

where

$$
\left.\beta \equiv \frac{\left\langle\left|\Phi_{z}\right|\right\rangle}{2}\right|_{z=0}
$$

is a parameter that depends on the shape of the buoyancy and velocity profiles at the interface and, relating to a buoyancy flux, does not depend explicitly on entrainment. As discussed in 4.1 the reasoning leading to 4.19 assumes that the Péclet number is sufficiently large for this Boussinesq flow to equate BPE production $\left\langle\Phi_{d}\right\rangle$ with APE dissipation $G$.

Buoyancy can only do work on the flow over a height $\zeta$; therefore, not more than $\zeta$ of the supply of APE from the plume source is converted into kinetic energy, which means that the total diapycnal mixing in the lower layer is

$$
\left\langle\left\langle\Phi_{d}\right\rangle\right\rangle_{-\zeta}^{0}=1-\left\langle\left\langle\Phi_{d}\right\rangle\right\rangle_{0}^{1-\zeta}-\zeta=(1-\zeta)(1-\beta)
$$

If the profiles for velocity and buoyancy in the plume are assumed to have a Gaussian form and temporal fluctuations are neglected then $\beta=1 / 4$, as demonstrated by equation (A 9) in appendix A. Consequently, for $\zeta=1 / 2$, equation 4.21 predicts that $3 / 8$ of the $\mathrm{APE}$ is converted into BPE in the lower layer, while $1 / 8$ of the APE is converted into $\mathrm{BPE}$ in the upper layer. As can be seen in the final two columns of table 2 , this prediction is consistent with observations from the domain with $S=4 / 3$ to within less than $1 \%$. The remaining $1 / 2$ of the supply of APE is converted into kinetic energy, as outlined in the previous section.

As supported by the observations reported in table 2, the mixing efficiency of this system, defined as $\left\langle\left\langle\Phi_{d}\right\rangle\right\rangle /\left(\langle\langle\varepsilon\rangle\rangle+\left\langle\left\langle\Phi_{d}\right\rangle\right\rangle\right)$ (Hughes et al. 2013 ) is equal to $1 / 2$ when $\zeta=1 / 2$. For other values of $\zeta$, the mixing efficiency would be equal to $1-\zeta$, which is consistent with the analysis of an emptying filling box (Davies Wykes et al. 2019). We note that $\zeta \neq 1 / 2$ could be manufactured in a quadrant of a closed domain by dividing the buoyancy flux from a single point source between multiple point sources (Linden et al. 1990) in 
one of the layers. However, if the mixing efficiency were then calculated over adjacent quadrants it would always be $1 / 2$, which suggests that the efficiencies that differ from $1 / 2$ in Davies Wykes et al. (2019) are associated with the open nature of the emptying filling box domain.

\subsection{A model for the vertical dependence of BPE production}

In this section we will derive a model for the scaling of the vertical flux of $\operatorname{APE}\left\langle w E_{a}\right\rangle$ with height and, consequently, the BPE production as a function of height. As outlined in the previous sections, the model is based on two layers of ambient fluid of infinite horizontal extent, leading to the reduced form of 4.3,

$$
\frac{\mathrm{d}}{\mathrm{d} z}\left\langle w E_{a}\right\rangle=-\left\langle\Phi_{d}\right\rangle+\left\langle\Phi_{z}\right\rangle
$$

which states that the destruction of $\operatorname{APE}\langle G\rangle \approx\left\langle\Phi_{d}\right\rangle$ balances the work done by buoyancy $\left\langle\Phi_{z}\right\rangle$ and differences in the boundary fluxes of APE.

Using Gaussian profiles for velocity and buoyancy, and 4.16 to evaluate $E_{a}$ leads to the following expression for the APE flux in the lower layer (see appendix A):

$$
\left\langle w E_{a}\right\rangle=1-\zeta Z-(1-\zeta) Z^{5 / 3}+\frac{1-\zeta}{4} Z^{10 / 3}, \quad 0 \leqslant Z<1,
$$

where $Z \equiv(z+\zeta) / \zeta$ is a rescaled vertical coordinate that measures the distance from the source as a proportion of the distance from the plume source to the interface $(Z=0$ at the source and $Z=1$ at the interface). In the lower layer, the relative buoyancy flux $-\left\langle\Phi_{z}\right\rangle$ is unity; hence, noting that $\zeta \mathrm{d} Z=\mathrm{d} z, 4.22$ indicates that

$$
\left\langle\Phi_{d}\right\rangle=\frac{5}{6} \frac{1-\zeta}{\zeta}\left(2 Z^{2 / 3}-Z^{7 / 3}\right), \quad 0 \leqslant Z<1 .
$$

At the interface, where $Z=1,4.24$ implies that

$$
\left.\left\langle\Phi_{d}\right\rangle\right|_{Z=1}=\frac{5}{6} \frac{1-\zeta}{\zeta} .
$$

In the upper layer, the APE dissipation and BPE production are equal to (minus) the vertical derivative of the APE flux:

$$
\langle G\rangle=\left\langle\Phi_{d}\right\rangle=-\frac{1-\zeta}{2} \frac{\mathrm{d}\left\langle\left|\Phi_{z}\right|\right\rangle}{\mathrm{d} z}>0, \quad z>0,
$$

using (4.17). Progress beyond 4.26 requires a model for the evolution of the APE flux in the upper layer, which depends on the rate at which the positively and negatively buoyant regions in the jet mix, as illustrated schematically in figure 13 . We develop a heuristic description of the process in terms of the mean buoyancy by assuming that the mixing of the core and the annular shell of the jet $(i)$ proceeds a rate that is proportional to the buoyancy deficit $b-b_{m}$. At the same time, the buoyancy deficit is reduced by $(i i)$ the overall dilution of the jet due to entrained ambient fluid. The dilution is accounted for by noting that $b-b_{m}$ is proportional to the flux of APE, $(1-\zeta)\left\langle\left|\Phi_{z}\right|\right\rangle / 2$, divided by a volume flux.

Provided that the virtual source of the jet coincides with that of the plume at $z=-\zeta$, the volume flux in the jet is proportional to $z+\zeta$, which is true for Gaussian jets and plumes because their spreading rates are almost identical (see equation (3.1) and van Reeuwijk \& Craske 2015); hence 


$$
\frac{\mathrm{d}\left\langle\left|\Phi_{z}\right|\right\rangle}{\mathrm{d} z}=-c \frac{\left\langle\left|\Phi_{z}\right|\right\rangle}{z+\zeta}, \quad z>0 .
$$

The processes of $(i)$ mixing and $(i i)$ overall dilution described above, and depicted in figure 13 , both relate to entrainment. Yet, as outlined at the start of this section, we do not expect an integral energy budget to require a closure for entrainment. With regards to $(i)$, it is known that the radial component of the turbulent transport of buoyancy is proportional to an entrainment coefficient in self-similar jets (in the case of passive scalars) and in plumes (Craske \& van Reeuwijk 2016). However, the entrained volume that is responsible for suppressing the rate at which process $(i)$ occurs is also proportional to an entrainment coefficient. Processes $(i)$ and $(i i)$ therefore combine in (4.27) to produce the parameter $c$ that can be deduced without explicit reference to an entrainment coefficient.

Using 4.27, 4.26) and ensuring $C^{0}$ continuity of $\left\langle\Phi_{d}\right\rangle$ at $z=0$, implies that

$$
c=\frac{5}{3 \zeta} \text {. }
$$

Integration of (4.27) and substitution of the result into 4.26 yields

$$
\left\langle\left|\Phi_{z}\right|\right\rangle=\frac{1}{2 Z^{c}}, \quad \text { and } \quad\left\langle\Phi_{d}\right\rangle=\frac{5}{12} \frac{1-\zeta}{\zeta^{2}} \frac{1}{Z^{c+1}} .
$$

For an interface at a distance $\zeta=1 / 2$ from the source, 4.29 implies that

$$
\left\langle\left|\Phi_{z}\right|\right\rangle=\frac{1}{2 Z^{10 / 3}}, \quad \text { and } \quad\left\langle\Phi_{d}\right\rangle=\frac{5}{6 Z^{13 / 3}}, \quad Z \geqslant 1 .
$$

The predictions obtained from (4.24) (lower layer, $0 \leqslant Z<1$ ) and 4.30) (upper layer, $1 \leqslant Z$ ) are included in figure $11(a)$. The upper layer model exhibits a reasonably good agreement with the simulation data for large aspect ratios, although the predicted value of $\Phi_{d}$ at the interface is relatively large. Indeed, the model assumed a step change in the ambient buoyancy rather than the more gradually varying profiles of buoyancy that are seen in the simulations (cf. figure 3p. It should also be noted that the model does not account for mixing induced by the horizontal boundaries because it does not include a length scale corresponding to the vertical confinement.

There appears to be a large difference between the observations and the model's predictions in the lower layer. However, the observations of $\Phi_{d}$ depend on aspect ratio and there are indications that the simulations exhibit an increasingly good agreement with the model's prediction as the aspect ratio increases. It is also worth noting that the model assumes a point source of buoyancy, rather than the finite circular source that was used in each simulation, leading to large differences between prediction and observation close to the source. On the largest domain, the existence of a local maximum in $\left\langle\Phi_{d}\right\rangle$ at approximately $z=-0.2$ is consistent with the model. We therefore suspect that observations of plumes emanating from smaller sources on domains of larger aspect ratio than those studied here would yield an improved agreement with the model.

\subsection{The radial dependence of velocity and buoyancy}

From an integral perspective, the heuristic arguments leading to the scaling described in 4.5 are reasonably consistent with the integral observations shown in figure 11 . However, the extent to which the integral model faithfully accounts for the underlying transport processes is unclear and is therefore the subject of this and the subsequent 
section, in which we study the radial dependence and vertical evolution of time and azimuthally averaged quantities.

Azimuthal averages were obtained by identifying computational cells that lie an equal distance from the vertical centre line of the two jets or plumes in a given layer (see, for example, the coordinates labelled ' $r$ ' in figure 1). We start by comparing the data to mean Gaussian profiles for velocity and buoyancy, derived in appendix A, whose predicted amplitude and width scale according to the model described in 4.5 . We note, however, that such a comparison is naive in relying on the assumption that the APE flux used in 4.5 is comprised entirely from mean flow processes, which we will demonstrate is not the case in 8.7 .

Figure 14 displays the radial dependence of the mean vertical velocity $\bar{w}(a, c, e)$ and mean buoyancy $\bar{b}(b, d, f)$ in the upper layer at heights $z=0.3(a, b) z=0.1(c, d)$ and $z=0.0(e, f)$ on domains of aspect ratio 1 and $4 / 3$. The observed profiles in figure 14 are rescaled using the model's predictions in terms of the rescaled distance $Z=(z+\zeta) / \zeta$ from the source, rather than being scaled arbitrarily to produce the best local agreement. The comparison therefore tests the ability of the model to predict the shape of the profiles, in addition to their evolution in the vertical direction.

The panels $(a),(c)$ and $(e)$ indicate that the velocity in the jet after the plume penetrates the stratification has an approximately Gaussian dependence on the radial coordinate. The similarity of the profiles in each panel suggest that the spatial evolution of $\bar{w}$ is indeed self-similar, and therefore confirms the explanation that was provided in 3.1 regarding the development of secondary circulation cells due to entrainment into the jets.

The model consistently over-estimates the maximum velocity, which is partly due to assignment of the total buoyancy flux input exclusively to mean transport, rather than turbulent transport. Whilst it is possible to account for the effects of turbulent transport in plume theory (Craske \& van Reeuwijk 2016), we refrain from doing so here to keep our approach as transparent as possible. It should also be noted that integrals over the horizontal cross-section of the flow are relatively insensitive to azimuthally averaged values close to the centre line $(r=0)$.

Relative to its local environment, the observed mean buoyancy displayed in $14(b, d, f)$ is positive in the core of the jet and negative in a shell starting at $r /\left(r_{m} Z\right) \gtrsim 0.5$ for heights $z=0.1$ and $z=0.3$, where $r_{m}=6 \alpha \zeta / 5$ is the radius of the plume at the interface. However, the amplitude of the observed mean buoyancy anomalies is significantly smaller than that predicted by theory. At the height of the interface $(z=0.0)$, the model predicts a step change in the ambient buoyancy, whereas the observed ambient buoyancy varies gradually (cf. figure 3). In this respect it is interesting that the observed mean buoyancy profile in panel $(f)$ is nowhere less than the ambient buoyancy at the interface. The buoyancy profile therefore only evidences the effects of a changing ambient for larger values of $z$. Unlike the mean velocity profiles, the average buoyancy profiles do not appear to be self-similar. For example, the shell of negatively buoyant fluid in $(b)$ at $z=0.3$ is relatively large in comparison with the equivalent region in panel $(d)$ at $z=0.1$.

It should be noted that the model developed in 4.5 does not permit a unique interpretation of the way in which the mean buoyancy returns to its ambient value when $r /\left(r_{m} Z\right) \rightarrow \infty$ at a given elevation. Outside the jet, the vertical velocity is negligible. Therefore, the mean buoyancy flux, which is the primary quantity on which the model is based, is also negligible, regardless of the precise form of the buoyancy profile.

The difference between theory and observation in the mean buoyancy profile shown in figure 14 raises the question of how the theory is able to correctly describe the integral scaling of BPE production in the upper layer of figure $11(a)$. The prediction of BPE 
$(a)$

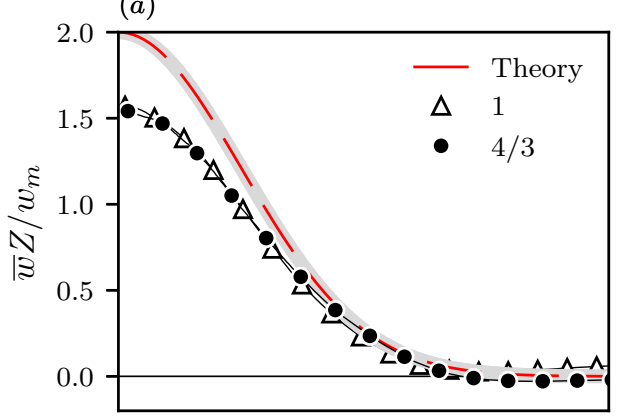

(c)

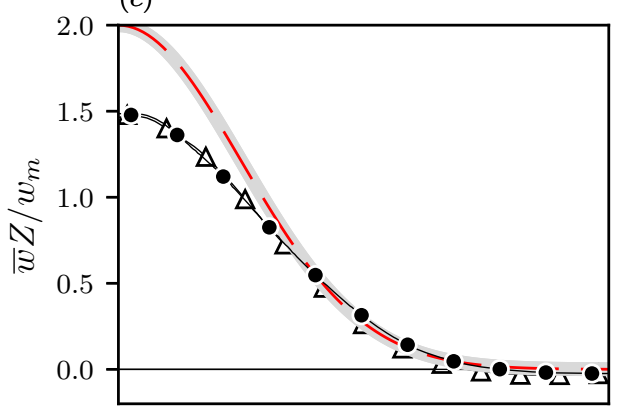

$(e)$

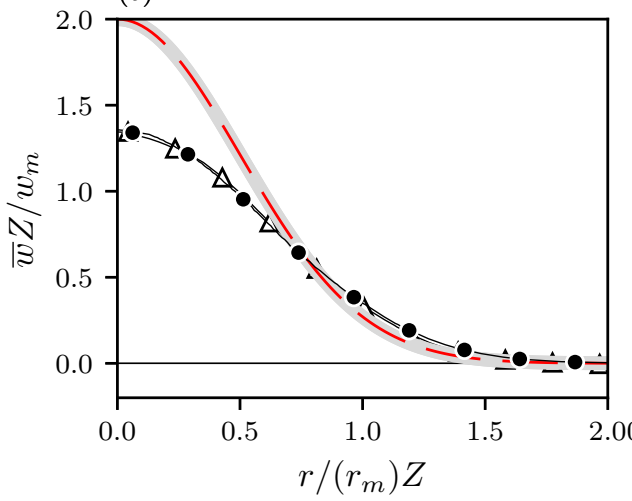

(b)

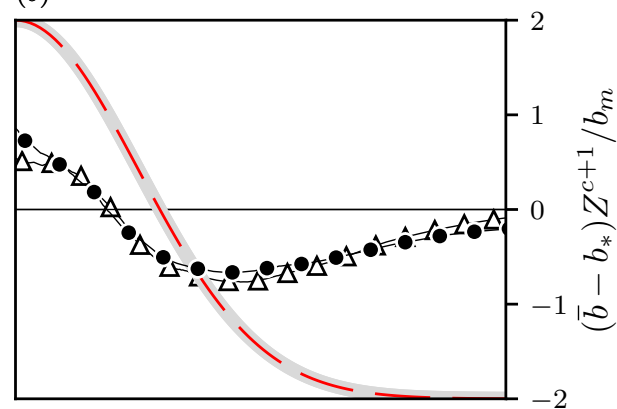

$(d)$

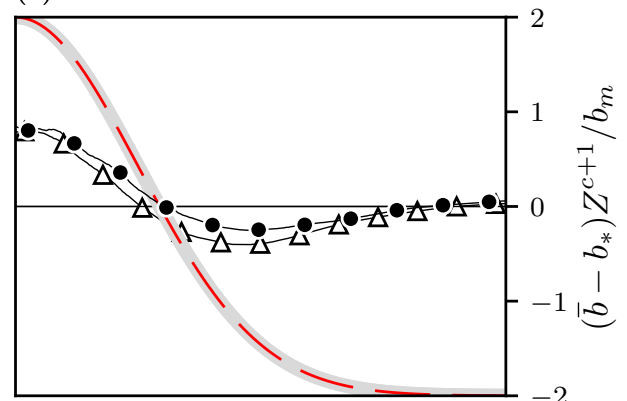

$(f)$

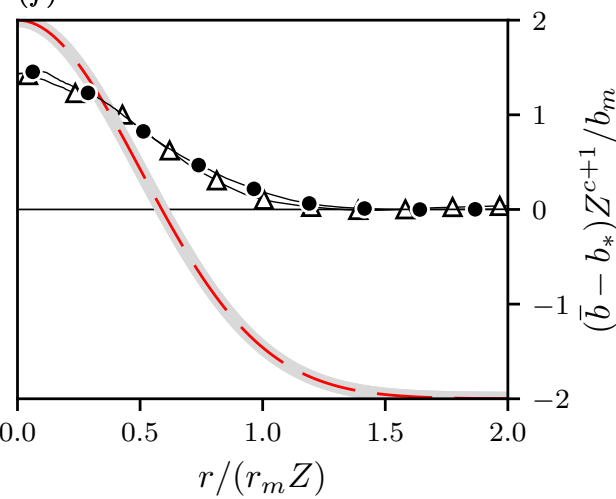

Figure 14. Mean velocity $\bar{w}$ (left column) and mean buoyancy in the jet, relative to the background state, $\bar{b}-b_{*}$ (right column) with respect to the radial similarity coordinate $r /\left(r_{m} Z\right)$, where $r_{m}=6 \alpha \zeta / 5$ is the radius of the plume at the interface and $Z$ is the distance from the source rescaled by the distance from the plume source to the interface $Z=(z+\zeta) / \zeta(=2 z+1$ for $\zeta=1 / 2)$ at heights $z=0.3(a, b), z=0.1(c, d)$ and $z=0.0(e, f)$. The symbols denote data corresponding to aspect ratios $S=1$ and $S=4 / 3$.

production requires an accurate description of APE transport, whose constituent physical mechanisms and radial distribution we therefore study in the following section.

\subsection{The turbulent transport of $A P E$}

Subject to the assumptions laid out in 4.4 the prediction of BPE production in a given control volume requires knowledge of boundary fluxes of APE. In this regard, the model described in 4.4 was motivated heuristically in terms of mean profiles of velocity and buoyancy (cf. figure 13). In a turbulent flow, however, both APE and its transport contain components that result from temporal fluctuations, which we study in 
(a)
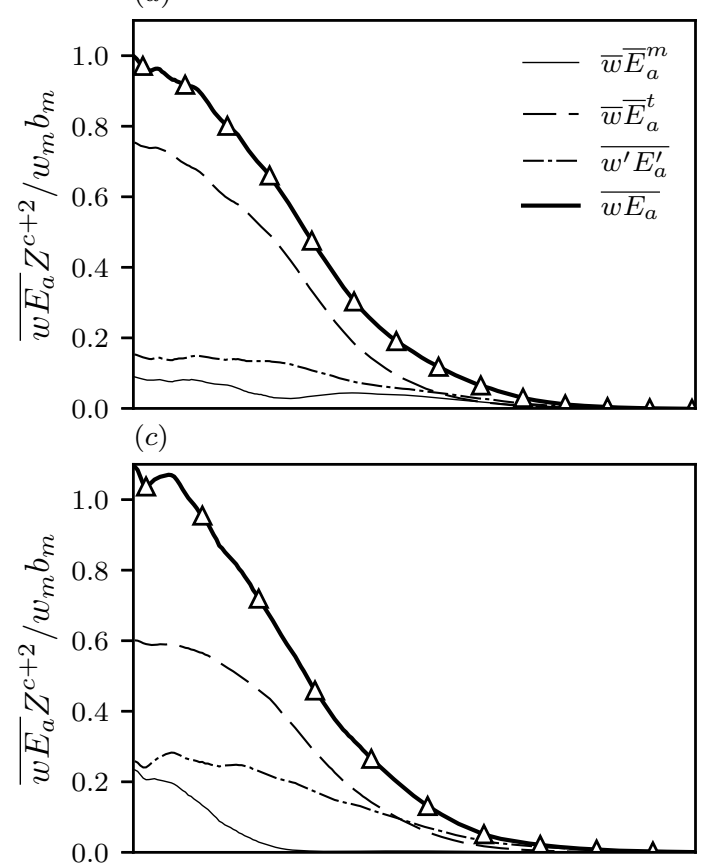

(e)

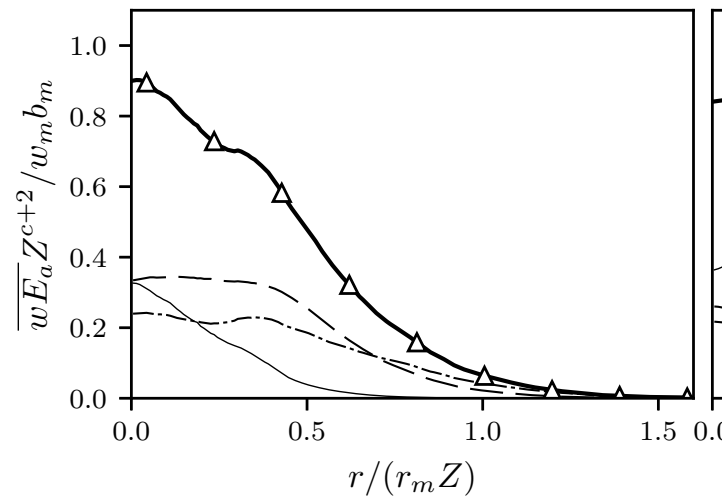

(b)
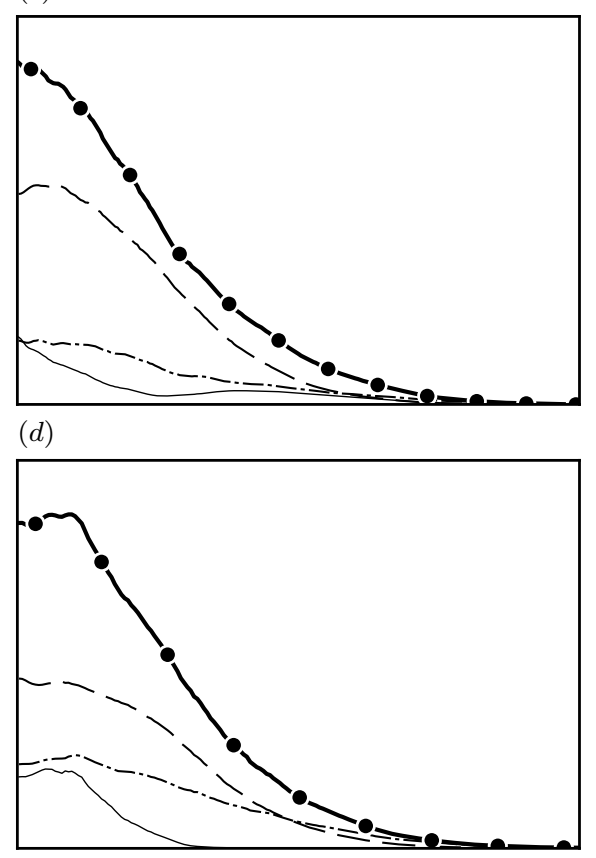

$(f)$

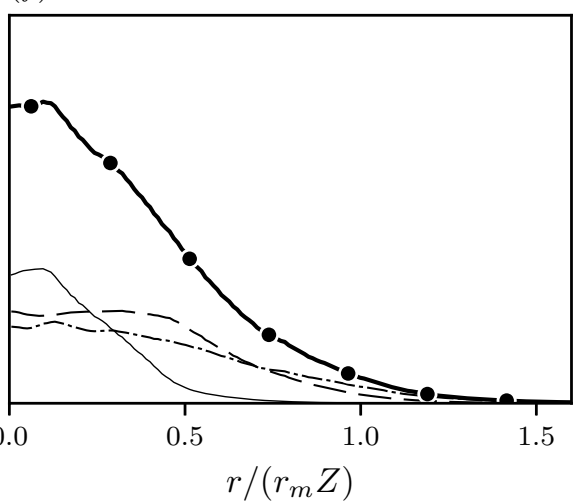

Figure 15. Observed profiles of the mean advective transport of APE $\overline{w E_{a}}=\bar{w} \bar{E}_{a}^{m}+\bar{w} \bar{E}_{a}^{t}+\overline{w^{\prime} E_{a}^{\prime}}$ with respect to the radial similarity coordinate $r /\left(r_{m} Z\right)$, where $Z$ is the distance from the source rescaled by the interface height $Z=(z+\zeta) / \zeta(=2 z+1$ as $\zeta=1 / 2)$ at heights $z=0.3(a, b), z=0.1(c, d)$ and $z=0.0(e, f)$. The left column corresponds to the aspect ratio $S=1$ and the right column corresponds to the aspect ratio $S=4 / 3$.

this section to explain the poor agreement between the prediction and observation of the mean buoyancy in figure 14

Due to the nonlinearity and convexity of $E_{a}(b)$, fluctuations $b^{\prime}=b-\bar{b}$ in the buoyancy field increase the mean APE $\bar{E}_{a}$. Following Scotti \& White (2014), it is therefore convenient to decompose the time-averaged APE into a function of the mean buoyancy field $\bar{E}_{a}^{m}=E_{a}(\bar{b})$ in addition with the APE arising from the fluctuations of the buoyancy $\bar{E}_{a}^{t}=\bar{E}_{a}-\bar{E}_{a}^{m}$, such that 


$$
E_{a}^{\prime} \equiv \underbrace{\int_{b_{*}}^{b}\left(z_{*}(\hat{b})-z\right) \mathrm{d} \hat{b}}_{E_{a}}-\underbrace{\int_{b_{*}}^{b}\left(z_{*}(\hat{b})-z\right) \mathrm{d} \hat{b}}_{\bar{E}_{a}^{m}+\bar{E}_{a}^{t}},
$$

defines temporal fluctuations in the APE whose mean $\overline{E_{a}^{\prime}}$ vanishes. Consequently, the average vertical transport of APE comprises three physically distinct processes, because

$$
\overline{w E_{a}}=\bar{w} \bar{E}_{a}^{m}+\bar{w} \bar{E}_{a}^{t}+\overline{w^{\prime} E_{a}^{\prime}}
$$

The first term on the right-hand side of equation 4.32 corresponds to transport by the mean vertical velocity $\bar{w}$ of the APE associated with the mean buoyancy $\bar{b}$. The second term corresponds to transport by the mean vertical velocity of the APE associated with fluctuations in the buoyancy, and the third term corresponds to transport by the turbulent velocity field. In general, fluctuations in the background state defined by $z_{*}$ would lead to additional terms. For further details the reader is referred to Scotti \& White (2014), who do not assume a steady background buoyancy field a priori.

Figure 15 displays the observed APE transport with respect to the similarity coordinate $r /\left(r_{m} Z\right)$ at heights $z=0.3(a, b), z=0.1(c, d)$ and $z=0.0(e, f)$ for aspect ratios $S=1$ $(a, c, e)$ and $S=4 / 3(b, d, f)$. The most significant feature of APE transport that can be seen in figure 15 is the dominant contribution from the advection of buoyancy fluctuations by the mean flow $\bar{w} \bar{E}_{a}^{t}$. In contrast, the APE flux resulting from mean profiles of velocity and buoyancy $\bar{w} \bar{E}_{a}^{m}$ is relatively insignificant, particularly for large values of $z$.

A second notable feature of figure 15 is that the profiles of the total APE flux $\overline{w E_{a}}$ are approximately self-similar, when scaled according to the theoretical model derived in \$4.5. However, the components 4.32 comprising $\overline{w E_{a}}$ are not individually self-similar, because their relative amplitude and shape changes with respect to $z$. Indeed, self-similar profiles of mean velocity and mean buoyancy will not produce self-similarity in $\bar{w} \bar{E}_{a}^{m}$; according to 4.16 the relative contribution of positively and negatively buoyant parts of the jet to $\bar{w} E_{a}^{n}$ depend on $z$ in different ways, which violates self-similarity. Some evidence of this can be found in figure 15, which shows that the profile of $\bar{w}_{a}^{m}$ becomes flatter as $z$ increases and one moves further from the interface.

We conclude that the heuristic arguments leading the scaling derived in $\$ 4.4$ should not be interpreted literally in terms of mean-flow profiles. In contrast with classical plume theory, from which fluctuating quantities are typically neglected, the fluctuating buoyancy field plays a dominant role in this flow's energetics via the flux $\bar{w} \bar{E}_{a}^{t}$. Whilst the notion that parcels of relatively dense fluid are dragged into the upper layer (cf. figure 13 is qualitatively correct, the process should be understood as leading to temporal fluctuations in the buoyancy field that are advected by the mean flow. In this regard it is particularly interesting that the flow organises itself to produce self-similarity in $\overline{w E_{a}}$, in spite of the evolving differences between the relative contributions from its constituent parts 4.32.

\section{Conclusions}

We have analysed the flow driven by equal and opposite point sources of buoyancy in closed domains. In $\$ 3$ we focused on the structure of the mean flow and buoyancy, whereas in 4 we focused on quantities pertaining to the system's energetics. These 
contrasting perspectives highlighted aspects of the problem that do and do not entail an explicit mathematical dependence on an entrainment coefficient, respectively. Unlike the strength of the resulting circulation and buoyancy differences studied in $\$ 3$, the vertical distribution of BPE production in $\$ 4$ (which was approximately equal to APE production in the cases we considered) was predicted without recourse to an entrainment coefficient. In this regard, entrainment determines a 'constitutive' relation between buoyancy (force) and volume flux (flow) that is not visible from the perspective of energetics when the power input to the system is fixed. An analogy in the context of turbulent RayleighBénard convection would be the relationship between the Nusselt number, buoyancy variance dissipation and the flow's energetics resulting from fixed buoyancy flux boundary conditions (cf. Hughes et al. 2013).

In the first part of this study we observed a stable two-layer stratification and three distinct types of steady-state circulation between the plumes on domains of sufficiently large aspect ratio. The primary and largest circulation cell extended over the full depth of the domain and corresponded to the transport of fluid between the layers via turbulent entrainment into the plumes. In each layer a secondary circulation cell, driven by entrainment into the jet-like flows adjacent to each plume, was observed. Between each secondary circulation, we observed a third circulation cell of relatively small vertical extent around the domain's mid-plane. We expect an understanding of such flow structures to be relevant in predicting the transport of airborne pollutants in confined environments. In this regard, we note that the single circulation cell found in domains of relatively small aspect ratio $(S \leqslant 15 / 24)$ can bifurcate to produce secondary circulation cells if the aspect ratio is increased (see, for example, figure 3). This observation suggests that assumptions about the mean or steady-state flow structure for a particular problem should be made with caution.

We compared two estimates of an effective entrainment coefficient for the system: one calculated from the volume flux at the domains' mid-plane and a second corresponding to the observed buoyancy difference between layers of constant buoyancy. For domains of relatively small aspect ratio, the two estimations were significantly different, due to the stratification of each layer and the effects of background turbulence. For domains of relatively large aspect ratio the two estimations coincided, but yielded an effective entrainment coefficient of 0.2 , which is notably higher than the typical value of 0.12 corresponding to an unconfined, non-interacting plume from a point source van Reeuwijk \& Craske 2015). A higher entrainment coefficient has implications for the design of ventilation systems that utilise stack-driven displacement ventilation, because the prediction of the height of the occupied layer depends on the assumed value for the entrainment coefficient. Whilst the use of an 'effective' virtual source (see e.g. Linden et al. 1990 ; Hunt \& Kaye 2001) can account for the relatively large entrainment coefficient, we would expect its position to depend on the intensity of background turbulence based on the trend exhibited in figure 6 of this study.

In the second part of this study we examined and modelled the system's energetics without invoking a closure to account for entrainment. Consistent with Hughes et al. (2013) and Gayen et al. (2013), we find a global mixing efficiency for this convective flow of 1/2. Our results are consistent with Davies Wykes et al. (2019), who demonstrate that the mixing efficiency of an emptying filling box is proportional to the relative depth of the upper layer. We extended their analysis by developing an analytical solution for Gaussian plume profiles. The production of BPE and dissipation of APE was consequently found to be three times larger in the layer containing the plume than the layer containing the jet, whereas the viscous dissipation of kinetic energy was divided equally between the two layers. In relation to our observations concerning entrainment, an understanding the 
system's energetics provides a logical step towards modelling the effect that background turbulence might have on plumes in confined environments.

We have examined confined plumes using both classical plume theory and an energetics framework. Consistent with the energetics that underpin Rayleigh-Bénard convection (Hughes et al. 2013), the dissipation of buoyancy variance which can be mathematically related to an entrainment coefficient (Craske et al. 2017), does not play a direct role in this system's energy budgets. We contrast this situation, for closed domains, with the result for open domains obtained by Davies Wykes et al. (2019). There, an expression for the mixing efficiency of an emptying filling box was found to depend on the interface height, which, due to the box's connection with an exterior, necessarily depends on the entrainment of volume into the plume.

As a first step towards the use of local APE frameworks to understand the physics behind entrainment, our hope is that the volume- and plane-averaged perspective developed in $\$ 4$ provides a useful point of reference and link with existing work in the context of plume modelling. Progress in this respect might come from closer scrutiny of the radial dependence of APE dissipation in a plume, particularly in the vicinity of the turbulent/non-turbulent interface.

\section{Acknowledgements}

J.C. gratefully acknowledges an Imperial College Junior Research Fellowship. Computational resources for this work came from an EPSRC ARCHER Leadership grant, the 'Cambridge Service for Data Driven Discovery' (CSD3, http://csd3.cam.ac.uk) system operated by the University of Cambridge Research Computing Service (http: //www.hpc.cam.ac.uk) funded by EPSRC Tier-2 capital grant EP/P020259/1 and the High Performance Computing facility at Imperial College London. We would also like to acknowledge helpful suggestions from four anonymous referees, particularly those concerning local APE frameworks.

\section{Appendix A. Evaluation of the APE flux}

Following classical plume theory under the assumption of Gaussian profiles for buoyancy and velocity, we assume that below the interface the dimensionless velocity and buoyancy profiles can be described by the functions

$$
b=4 b_{m} \exp \left(-2 \eta^{2}\right) Z^{-5 / 3}-b_{m}, \quad w=2 w_{m} \exp \left(-2 \eta^{2}\right) Z^{-1 / 3},
$$

where $Z \equiv(z+\zeta) / \zeta$ is the distance from the source rescaled by the interface height, $\eta=r /\left(r_{m} Z\right)$ is a similarity coordinate based on the radial distance from the axis of the plume (see figure 1), $r_{m}=6 \alpha \zeta / 5$ is the radius of the plume at the interface and

$$
w_{m}=\frac{5}{6 \alpha}\left(\frac{9 \alpha}{10 \pi}\right)^{1 / 3} \zeta^{-1 / 3},
$$

is a characteristic velocity at the interface, such that $Q_{m}=\pi w_{m} r_{m}^{2}$.

Assuming a step change from $-b_{m}$ to $b_{m}$ in the ambient buoyancy across the interface, the buoyancy just above the interface is:

$$
b=\left(4 b_{m} \exp \left(-2 \eta^{2}\right)-2 b_{m}\right) Z^{-c-1}+b_{m}
$$


where $c=10 / 3$, as derived in $\$ 4.5$. The velocity in the upper layer is assumed to scale according to a turbulent jet:

$$
w=2 w_{m} \exp \left(-2 \eta^{2}\right) Z^{-1} .
$$

As required, the profiles (A 1), A 3 and A 4 are such that the relative buoyancy flux

$$
2 \pi \int_{0}^{\infty} w\left(b-b_{*}\right) r \mathrm{~d} r= \begin{cases}1, & z<0, Z<1, b_{*}=-b_{m} \\ 0, & z>0, Z>1, b_{*}=b_{m}\end{cases}
$$

A.1. Evaluation of $\left\langle w E_{a}\right\rangle$ below the interface $Z<1, z<0$

To evaluate the integral of the APE flux $w E_{a}$ below the interface we substitute A 1 ) into 4.16 and integrate over the area of a single plume. To account for $z_{*}(b)$, which 4.15 indicates is discontinuous with respect to $b$, it is useful to recognise that $b=b_{m}$ in $\sqrt{\mathrm{A} 1}$ 1 $a$ ) when $\eta=\eta_{0}=\sqrt{\log \left(2 Z^{-5 / 3}\right) / 2}$ and split the resulting integral into two parts. Noting that $2 \pi w_{m} b_{m} r_{m}^{2}=1$ is the buoyancy flux,

$$
\begin{aligned}
2 \pi r_{m}^{2} Z^{2} \int_{0}^{\eta_{0}} w E_{a} \eta \mathrm{d} \eta & =1-\zeta Z-(1-\zeta) Z^{5 / 3}+\frac{1-2 \zeta}{4} Z^{10 / 3}+\frac{\zeta}{4} Z^{13 / 3}, \\
2 \pi r_{m}^{2} Z^{2} \int_{\eta_{0}}^{\infty} w E_{a} \eta \mathrm{d} \eta & =\frac{\zeta}{4} Z^{10 / 3}-\frac{\zeta}{4} Z^{13 / 3},
\end{aligned}
$$

such that

$$
\left\langle w E_{a}\right\rangle=1-\zeta Z-(1-\zeta) Z^{5 / 3}+\frac{1-\zeta}{4} Z^{10 / 3}
$$

\section{A.2. Evaluation of $\left\langle w E_{a}\right\rangle$ above the interface $Z>1, z>0$}

As explained in $\$ 4.5$, above the interface

$$
\left\langle w E_{a}\right\rangle=(1-\zeta) \frac{\left\langle\left|\Phi_{z}\right|\right\rangle}{2}=(1-\zeta) \frac{\left\langle\left|w\left(b-b_{m}\right)\right|\right\rangle}{2} .
$$

The integral of $\left|\Phi_{z}\right|$ can be evaluated by integrating $w\left(b_{m}-b\right)>0$ over the shell in which $b<b_{m}$. According to (A 3), $\eta_{0}=\sqrt{\log (2) / 2}$ defines the point at which $b=b_{m}$ when $Z \geqslant 1$; hence, noting again that $2 \pi w_{m} b_{m} r_{m}^{2}=1$,

$$
\frac{\left\langle\left|\Phi_{z}\right|\right\rangle}{2}=\frac{1}{Z^{c}} \int_{\eta_{0}}^{\infty}\left(\exp \left(-2 \eta^{2}\right)-2 \exp \left(-4 \eta^{2}\right)\right) \eta \mathrm{d} \eta=\frac{1}{4 Z^{c}} .
$$

At the interface $Z=1$ and $(1-\zeta)\left\langle\left|\Phi_{z}\right|\right\rangle / 2=1 / 8$, which is consistent with (A 7). As $\beta$ is defined as $\left\langle\left|\Phi_{z}\right|\right\rangle / 2$ evaluated at $Z=1$, A 9 shows that $\beta=1 / 4$ for a plume with Gaussian velocity and buoyancy profiles.

\section{Appendix B. Validation}

To validate our findings, we ran each of the simulations reported in the main text with a reduced grid resolution, as described in $\mathrm{B} .1$. In $\mathrm{B} .2$, we describe simulations that 
were run to check the sensitivity of the flow structures discussed in $\$ 3$ with respect to a reduction in the Reynolds number.

\section{B.1. Grid resolution}

To verify the independence of our results to grid resolution we ran each of the simulations listed in table 1 using half as many computational cells in each spatial direction. Specifically, we employed 384 cells in the vertical $(z)$ direction and $384,438,480,512,768,1024$ cells in the horizontal directions for quadrant aspect ratios $1 / 2,7 / 12,15 / 24,2 / 3,1$, and $4 / 3$, respectively.

The quantities displayed in figure 16 , corresponding to those of figure 3 , indicate a good agreement between the results obtained from each of the two computational grids. The coarser grids reproduce the difference in buoyancy between the upper and lower layers (figure 16 $(a, d, g, j, m, p)$ ), in addition to the primary, secondary and tertiary circulation cells discussed in $\$ 3$. With regards to the latter, we note that the results of the original simulations were not used as initial conditions for the coarser grids, and therefore regard the existence of the circulation patterns reported in $\$ 3$ as robust for the parameters used in this study.

\section{B.2. Reynolds number}

To observe the local effect of changes in Reynolds number to the simulation results, we reduced the Reynolds number from 4185 to 2929, whilst employing the coarser computational grid described in B.1. The results shown in figure 17 suggest that the reduction in Reynolds does not cause a qualitative change in our findings regarding the internal temperature structure and circulation patterns. In particular, at $R e=2929$ the plumes are turbulent and, based on there being no observed change in the temperature difference between upper and lower layers in figure $17(p)$, entrain at approximately the same rate as when $R e=4185$. The results suggest that the existence of primary, secondary and tertiary circulation cells are robust to relatively small changes in Reynolds number, notwithstanding differences in the maximum volume flux seen in panels $(e, S=$ $7 / 12)$ and $(q, S=4 / 3)$. In the case of $(e, S=7 / 12)$, the quadrant volume flux depends sensitively on aspect ratio and on the slowly-varying organisation of the flow, which makes the precise determination of Reynolds number sensitivity difficult. Therefore, without data corresponding to additional aspect ratios for a larger range of Reynolds numbers, we note that the precise aspect ratios at which bifurcations in the mean flow between quadrants occur could depend on Reynolds number.

\section{REFERENCES}

Andrews, D. G. 1981 A note on potential energy density in a stratified compressible fluid. Journal of Fluid Mechanics 107, 227-236.

Baines, W. D. \& Turner, J. S. 1969 Turbulent buoyant convection from a source in a confined region. Journal of Fluid Mechanics 37 (1), 51-80.

Batchelor, G. K. 1954 Heat convection and buoyancy effects in fluids. Quarterly Journal of the Royal Meteorological Society 80 (345), 339-358.

Bonnebaigt, R., Caulfield, C. P. \& Linden, P. F. 2018 Detrainment of plumes from vertically distributed sources. Environmental Fluid Mechanics 18 (1), 3-25.

Burridge, H. C., Parker, D. A., Kruger, E. S., Partridge, J. L. \& Linden, P. F. 2017 Conditional sampling of a high Péclet number turbulent plume and the implications for entrainment. Journal of Fluid Mechanics 823, 26-56.

Camassa, R., Lin, Z., Mclaughlin, R. M., Mertens, K., Tzou, C., Walsh, J. \& White, B. 2016 Optimal mixing of buoyant jets and plumes in stratified fluids: theory and experiments. Journal of Fluid Mechanics 790, 71-103. 

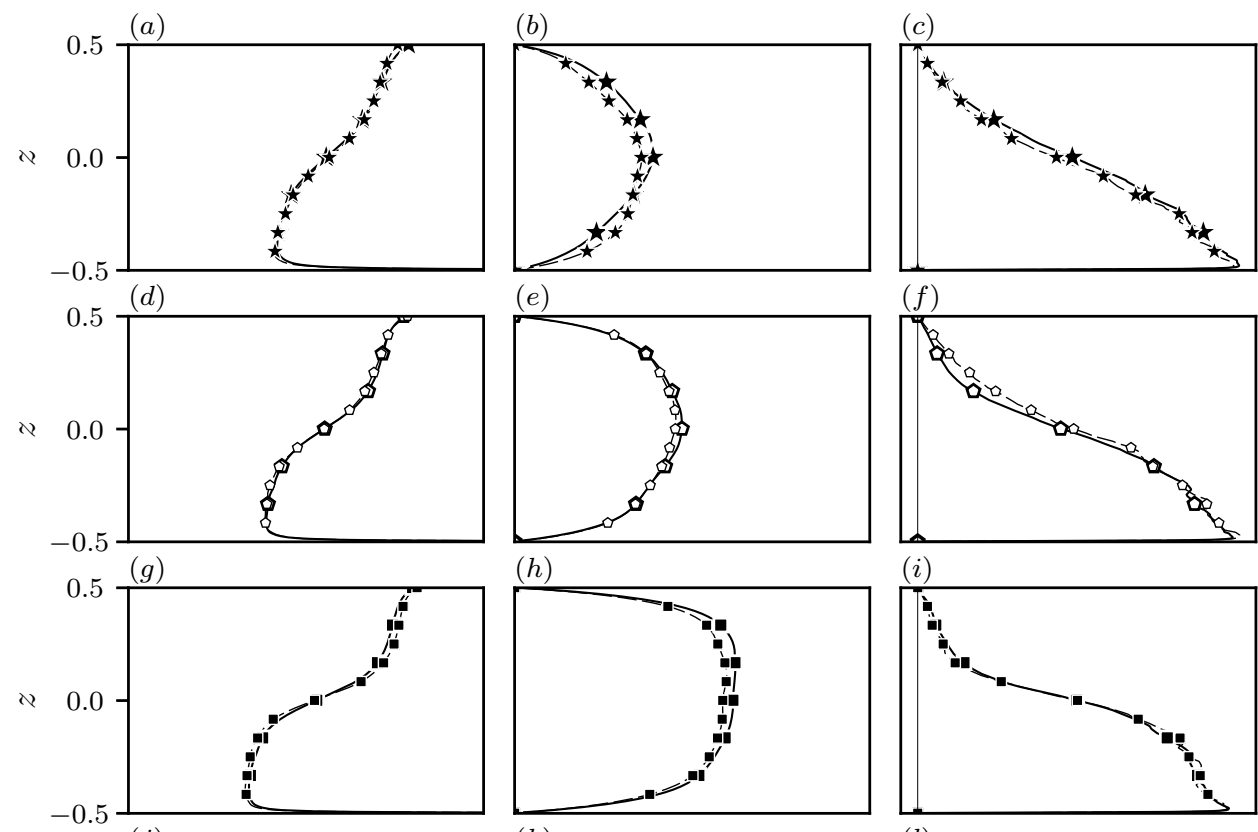

$(h)$
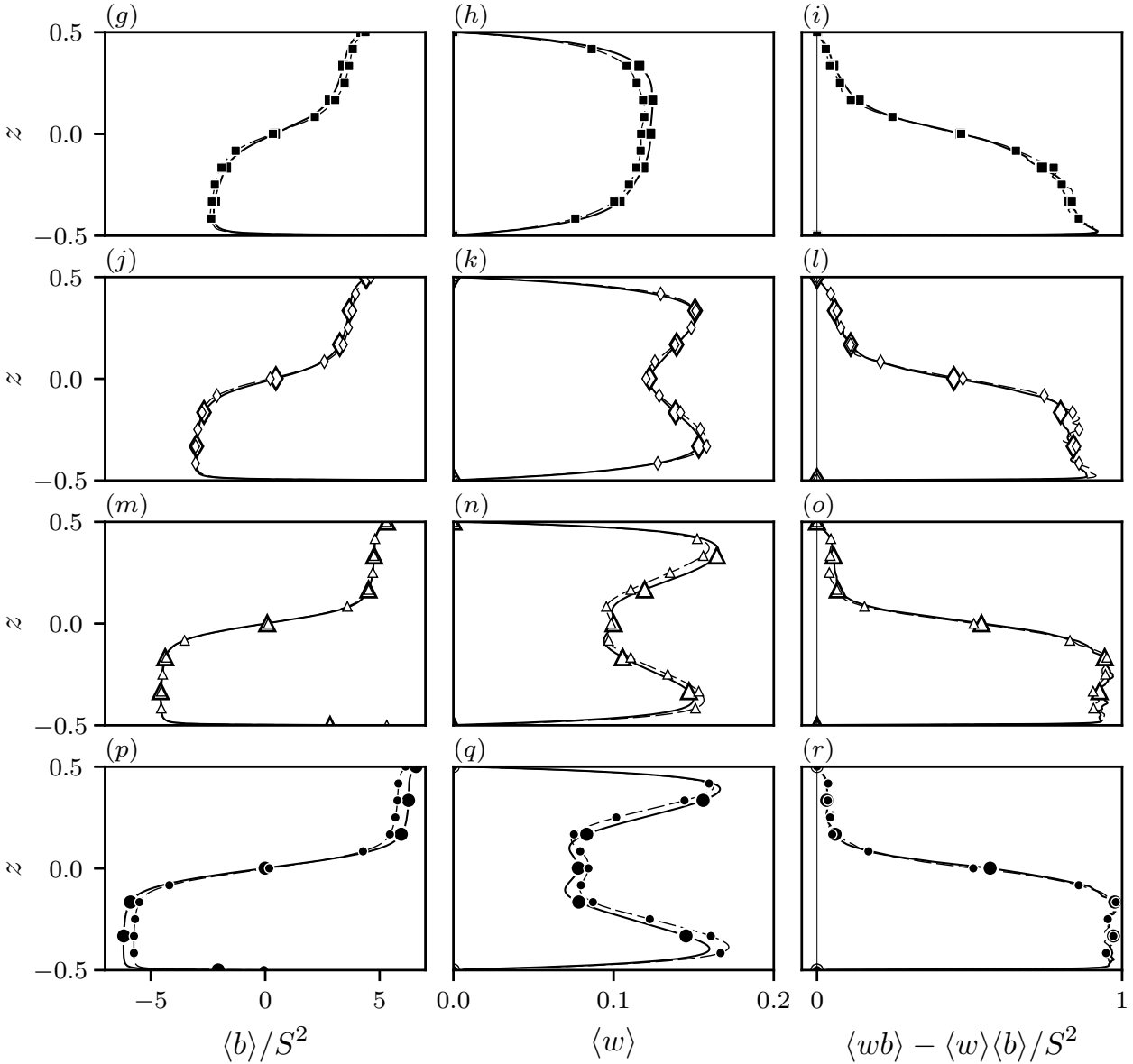

Figure 16. Grid resolution study. Horizontally- and time-averaged buoyancy (first column), with quadrant integrals of volume flux (second column) and relative buoyancy flux (third column) for aspect ratios $S=1 / 2,7 / 12,15 / 24,2 / 3,1$, and $4 / 3$, from top row to bottom row, respectively. The symbols correspond to those defined in table 1 and used in figure 3 . The dashed lines and relatively small symbols correspond to results obtained at the full grid resolutions reported in table 1 . The solid lines and relatively large symbols correspond to results obtained from grids employing half as many grid cells as those reported in table 1 in each spatial direction. 

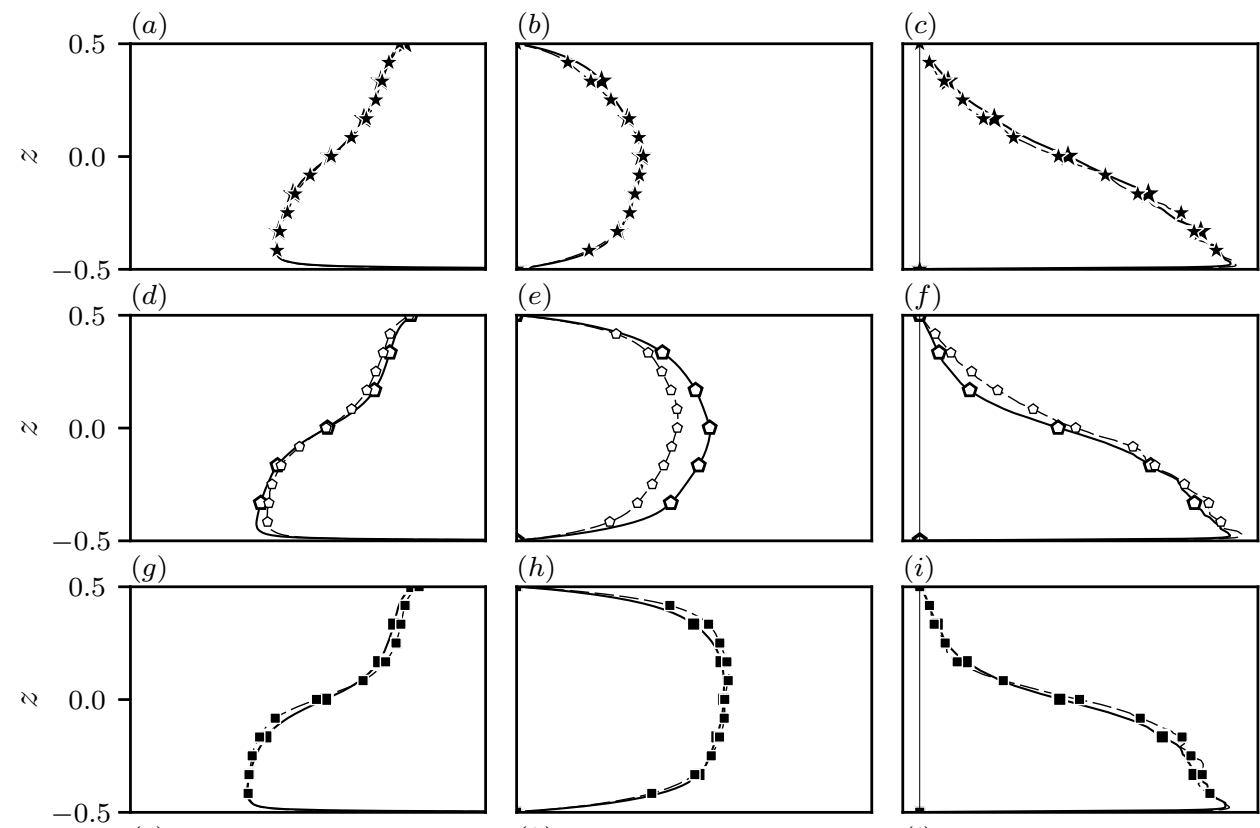

$(h)$
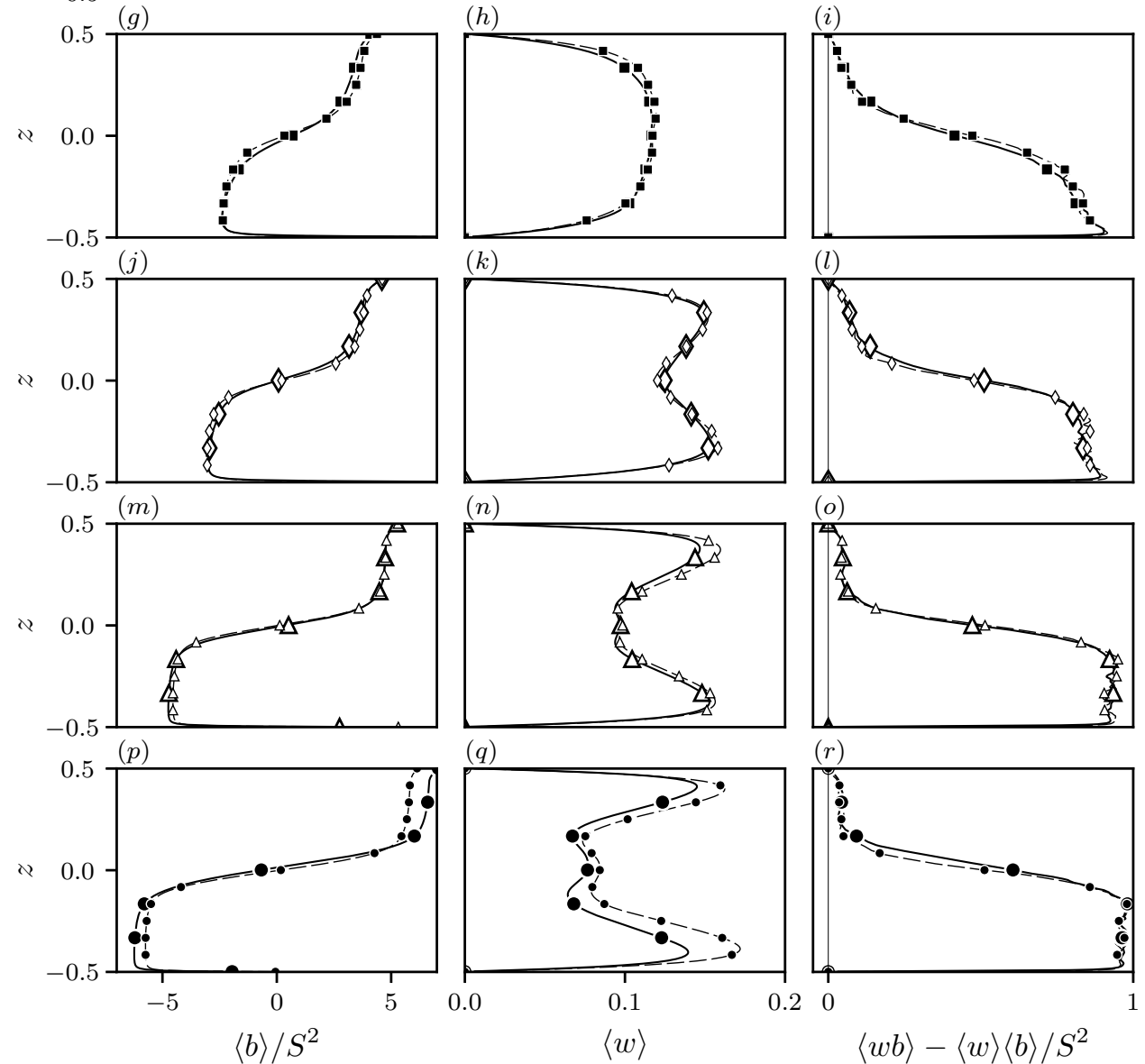

Figure 17. Reynolds number study. Horizontally- and time-averaged buoyancy (first column), with quadrant integrals of volume flux (second column), and relative buoyancy flux (third column) for aspect ratios $S=1 / 2,7 / 12,15 / 24,2 / 3,1$, and $4 / 3$, from top row to bottom row, respectively. The comparison is between results for $R e=4185$ (dashed lines and small symbols) using the grids reported in table 1 and those for $R e=2929$ (solid lines and large symbols) using coarse grids, comprising half as many points as those in table 1 in each spatial direction. 
Carazzo, G., Kaminski, E. \& Tait, S. 2006 The route to self-similarity in turbulent jets and plumes. Journal of Fluid Mechanics 547, 137-148.

Craske, J. \& van Reeuwijk, M. 2015 a Energy dispersion in turbulent jets. Part 1. Direct simulation of steady and unsteady jets. Journal of Fluid Mechanics 763, 500-537.

Craske, J. \& van ReeuniJk, M. $2015 b$ Energy dispersion in turbulent jets. Part 2. A robust model for unsteady jets. Journal of Fluid Mechanics 763, 538-566.

Craske, J. \& van ReeuwiJk, M. 2016 Generalised unsteady plume theory. Journal of Fluid Mechanics 792, 1013-1052.

Craske, J., Salizzoni, P. \& van Reeuwijk, M. 2017 The turbulent Prandtl number in a pure plume is 3/5. Journal of Fluid Mechanics 822, 774-790.

Davies Wykes, M. S., Hogg, C., Partridge, J. \& Hughes, G. O. 2019 Energetics of mixing for the filling box and the emptying-filling box. Environmental Fluid Mechanics 19 (4), 819-831.

FAnneløp, T. \& WebBer, D. M. 2003 On buoyant plumes rising from area sources in a calm environment. Journal of Fluid Mechanics 497, 319-334.

Gayen, B., Hughes, G. O. \& Griffiths, R. W. 2013 Completing the mechanical energy pathways in turbulent Rayleigh-Bénard convection. Physical Review Letters 111 (12), 124301.

Gladstone, C. \& Woods, A. W. 2014 Detrainment from a turbulent plume produced by a vertical line source of buoyancy in a confined, ventilated space. Journal of Fluid Mechanics 742, 35-49.

Gregg, M., D'Asaro, E., Riley, J. \& Kunze, E. 2018 Mixing efficiency in the ocean. Annual Review of Marine Science 10 (1), 443-473, pMID: 28934598.

Grossmann, S. \& Lohse, D. 2000 Scaling in thermal convection: a unifying theory. Journal of Fluid Mechanics 407, 27-56.

Holliday, D. \& Mcintyre, M. E. 1981 On potential energy density in an incompressible, stratified fluid. Journal of Fluid Mechanics 107, 221-225.

Hubner, J. 2006 Buoyant plumes in a turbulent environment. PhD thesis, University of Cambridge.

Hughes, G. O., Gayen, B. \& Griffiths, R. W. 2013 Available potential energy in RayleighBérnard convection. Journal of Fluid Mechanics 729, R3.

Hunt, G. R. \& Kaye, N. B. 2005 Lazy plumes. Journal of Fluid Mechanics 533, 329-338.

Hunt, G. R. \& Kaye, N. G. 2001 Virtual origin correction for lazy turbulent plumes. Journal of Fluid Mechanics 435, 377-396.

Hunt, J. C. R., Eames, I. \& Westerweel, J. 2006 Mechanics of inhomogeneous turbulence and interfacial layers. Journal of Fluid Mechanics 554, 499-519.

Kaminski, E., Tait, S. \& Carazzo, G. 2005 Turbulent entrainment in jets with arbitrary buoyancy. Journal of Fluid Mechanics 526, 361-376.

Khorsandi, B., Gaskin, S. \& Mydlarski, L. 2013 Effect of background turbulence on an axisymmetric turbulent jet. Journal of Fluid Mechanics 736, 250-286.

Lai, A. C. H., Law, A. W.-K. \& AdAms, E. E. 2019 A second-order integral model for buoyant jets with background homogeneous and isotropic turbulence. Journal of Fluid Mechanics 871, 271-304.

Linden, P. F. 1999 The fluid mechanics of natural ventilation. Annual Review of Fluid Mechanics 31 (1), 201-238.

Linden, P. F., Lane-Serff, G. F. \& Smeed, D. A. 1990 Emptying filling boxes: the fluid mechanics of natural ventilation. Journal of Fluid Mechanics 212, 309-335.

Lorenz, E. 1955 Available potential energy and the maintenance of the general circulation. Tellus 7 (2), 157-167.

Morton, B. R., Taylor, G. I. \& Turner, J. S. 1956 Turbulent gravitational convection from maintained and instantaneous sources. Proceedings of the Royal Society of London. Series A. Mathematical and Physical Sciences 234 (1196), 1-23.

Novak, L. \& Tailleux, R. 2018 On the local view of atmospheric available potential energy. Journal of the Atmospheric Sciences 75 (6), 1891-1907.

Priestley, C. H. B. \& BAll, F. K. 1955 Continuous convection from an isolated source of heat. Quarterly Journal of the Royal Meteorological Society 81 (348), 144-157. 
van Reeuwijk, M. \& Craske, J. 2015 Energy-consistent entrainment relations for jets and plumes. Journal of Fluid Mechanics 782, 333-355.

van ReeuwiJk, M. \& Holzner, M. 2014 The turbulence boundary of a temporal jet. Journal of Fluid Mechanics 739, 254-275.

van Reeuwijk, M., Salizzoni, P., Hunt, G. R. \& Craske, J. 2016 Turbulent transport and entrainment in jets and plumes: A DNS study. Phys. Rev. Fluids 1, 074301.

Roullet, G. \& Klein, P. 2009 Available potential energy diagnosis in a direct numerical simulation of rotating stratified turbulence. Journal of Fluid Mechanics 624, 45-55.

Scotti, A., Beardsley, R. \& Butman, B. 2006 On the interpretation of energy and energy fluxes of nonlinear internal waves: an example from massachusetts bay. Journal of Fluid Mechanics 561, 103-112.

Scotti, A. \& White, B. 2014 Diagnosing mixing in stratified turbulent flows with a locally defined available potential energy. Journal of Fluid Mechanics 740, 114-135.

da Silva, C. B., Hunt, J. C. R., Eames, I. \& Westerweel, J. 2014 Interfacial layers between regions of different turbulence intensity. Annual Review of Fluid Mechanics 46 (1), 567-590.

Tailleux, R. 2009 On the energetics of stratified turbulent mixing, irreversible thermodynamics, Boussinesq models and the ocean heat engine controversy. Journal of Fluid Mechanics 638, 339-382.

TAilleux, R. 2018 Local available energetics of multicomponent compressible stratified fluids. Journal of Fluid Mechanics 842, R1.

TAYlOR, G. I. 1958 Flow induced by jets. J. Aero./Space Sci. 25, 464-465.

Winters, K. B., Lombard, P. N., Riley, J. J. \& D'Asaro, E. A. 1995 Available potential energy and mixing in density-stratified fluids. Journal of Fluid Mechanics 289, 115-128.

Worster, M. G. \& Huppert, H. E. 1983 Time-dependent density profiles in a filling box. Journal of Fluid Mechanics 132, 457-466. 